\title{
CONTAM Results Export Tool
}

Brian Polidoro

Lisa C. $\mathrm{Ng}$

W. Stuart Dols

\section{CONTAM Results Export Tool}

Select an export option.

- Export .sim file to text files

Compute temporal/spatial averages of concentrations

Process Exposure Results

Export infiltration to EnergyPlus: whole building air change rates

Export infiltration to EnergyPlus: zone-specific air change rates

\section{Next}

Version 3.2

This publication is available free of charge from: https://doi.org/10.6028/NIST.TN.1912r1

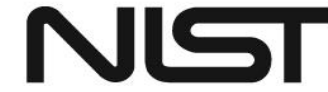

National Institute of Standards and Technology

U.S. Department of Commerce 


\title{
NIST Technical Note 1912 \\ Revision 1
}

\section{CONTAM Results Export Tool}

\author{
Brian Polidoro \\ Lisa C. $\mathrm{Ng}$ \\ W. Stuart Dols \\ Energy and Environment Division \\ Engineering Laboratory
}

This publication is available free of charge from:

https://doi.org/10.6028/NIST.TN.1912r1

April 2021

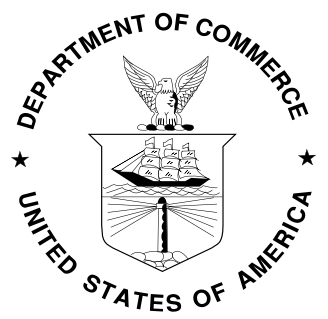

U.S. Department of Commerce Gina M. Raimondo, Secretary

National Institute of Standards and Technology James K. Olthoff, Performing the Non-Exclusive Functions and Duties of the Under Secretary of Commerce for Standards and Technology \& Director, National Institute of Standards and Technology 
Certain commercial entities, equipment, or materials may be identified in this document in order to describe an experimental procedure or concept adequately. Such identification is not intended to imply recommendation or endorsement by the National Institute of Standards and Technology, nor is it intended to imply that the entities, materials, or equipment are necessarily the best available for the purpose.

National Institute of Standards and Technology Technical Note 1912 Revision 1 Natl. Inst. Stand. Technol. Tech. Note 1912, 33 pages (April 2021) CODEN: NTNOEF

This publication is available free of charge from: https://doi.org/10.6028/NIST.TN.1912r1 


\begin{abstract}
CONTAM is a multizone indoor air quality and ventilation analysis program developed by the National Institute of Standards and Technology (NIST) (Dols and Polidoro 2015). CONTAM generates a wide range of different simulation outputs in the form of binary and text-based files. CONTAM provides the ability to view a limited set of these results via the CONTAM graphical user interface, but many users require access to a much broader range of results. The CONTAM Results Export Tool (subsequently referred to as "Export Tool") was created to provide access to all of the results that can be generated by a CONTAM simulation. It does so by reading the binary result files and converting them to tab-delimited text files that can be read using a spreadsheet program. The results that can be exported include contaminant concentrations; zone temperatures; weather conditions; airflows through individual paths, ducts and duct leakages; temporal and spatial averages of zone concentrations; and exposure results. The Export Tool also includes the ability to export infiltration rates for use in simulations performed with the EnergyPlus whole-building energy simulation program. This document describes five features of the Export Tool: (1) general conversion of binary results to text, (2) computing temporal and spatial averages of zone contaminant concentrations, (3) exporting occupant exposure results, (4) exporting whole-building air change rates from a CONTAM simulation for use in EnergyPlus, and (5) exporting zone-specific air change rates from a CONTAM simulation for use in EnergyPlus. The Export Tool is available, along with CONTAM and other NISTdeveloped software, on the NIST Multizone Modeling Website http://www.bfrl.nist.gov/IAQanalysis.
\end{abstract}

\title{
Keywords
}

airflow, CONTAM, contaminants, occupant exposure, simulation 


\section{Table of Contents}

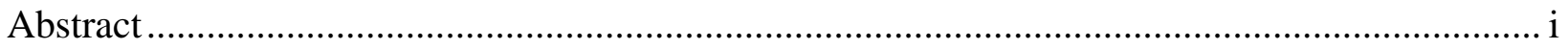

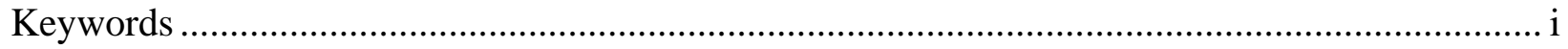

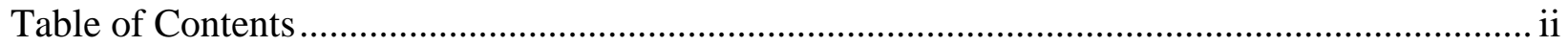

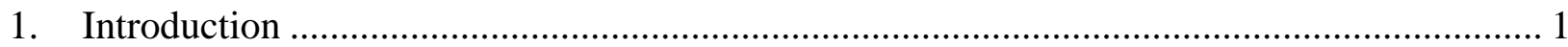

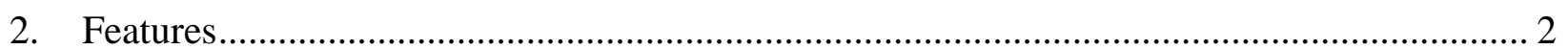

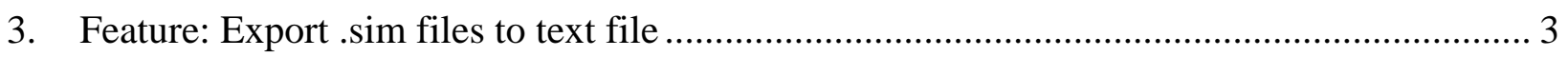

3.1. Step-by-step instructions for the "Export sim files to text file" feature ............................ 4

4. Feature: Compute temporal/spatial averages of concentrations ........................................... 9

4.1. Step-by-step instructions for the "Compute temporal/spatial averages of concentrations"

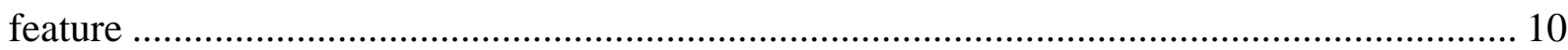

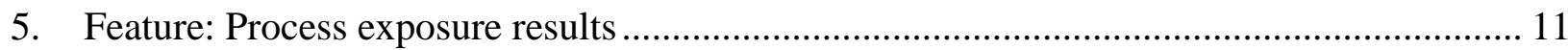

5.1. Step-by-step instructions for the "Process exposure results" feature.............................. 12

6. Feature: Export infiltration to EnergyPlus: whole-building air change rates ......................... 13

6.1. Background: Modeling infiltration in EnergyPlus ..................................................... 13

6.2. Limitations and Other Considerations......................................................................... 14

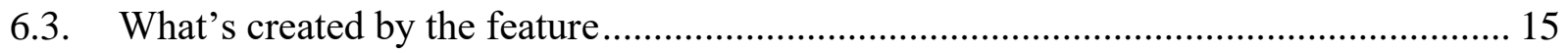

6.4. Using the feature ………………........................................................................ 17

6.5. Step-by-step instructions for the "Export infiltration to EnergyPlus: whole-building air

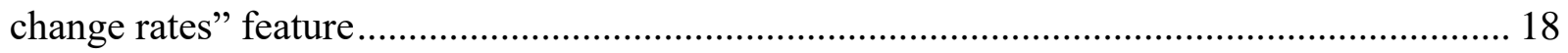

6.6. What to do with the .csv and partial idf files ......................................................... 19

7. Feature: Export infiltration to EnergyPlus: zone-specific air change rates ........................... 25

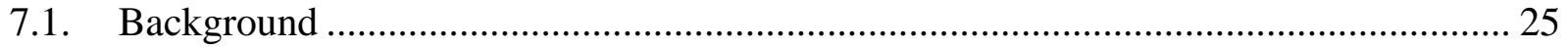

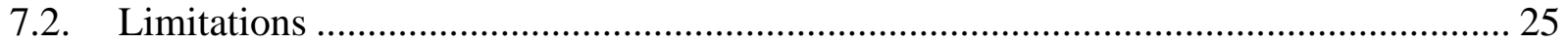

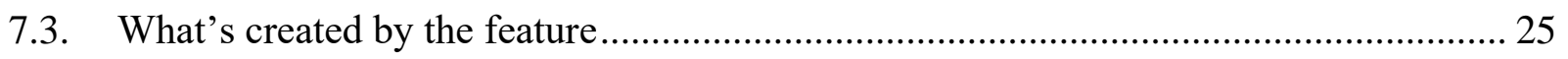



7.5. Step-by-step instructions for the "Export infiltration to EnergyPlus: zone-specific air



7.6. What to do with the .csv and partial idf files ....................................................... 29

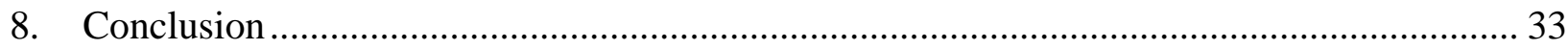

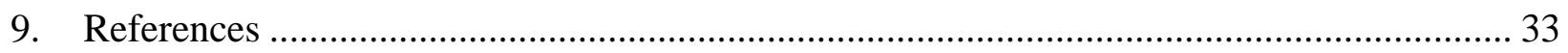




\section{Introduction}

CONTAM is a multizone indoor air quality and ventilation analysis program developed by the National Institute of Standards and Technology (NIST) (Dols and Polidoro 2015). CONTAM generates a wide range of different simulation outputs in the form of binary and text-based files. CONTAM provides the ability to view a limited set of these results via the CONTAM graphical user interface, but many users require access to a much broader range of results. The CONTAM Results Export Tool (subsequently referred to as "Export Tool") was created to provide access to all of the results that can be generated by a CONTAM simulation. It does so by reading the binary result files and converting them to tab-delimited text files that can be read using a spreadsheet program. The results that can be exported include contaminant concentrations; zone temperatures; weather conditions; airflows through individual paths, ducts and duct leakages; temporal and spatial averages of zone concentrations; and exposure results. The Export Tool also includes the ability to export infiltration rates for use in simulations performed with the EnergyPlus whole-building energy simulation program. This document describes five features of the Export Tool: (1) general conversion of binary results to text, (2) computing temporal and spatial averages of zone contaminant concentrations, (3) exporting occupant exposure results, (4) exporting whole-building air change rates from a CONTAM simulation for use in EnergyPlus, and (5) exporting zone-specific air change rates from a CONTAM simulation for use in EnergyPlus. The Export Tool is available, along with CONTAM and other NISTdeveloped software, on the NIST Multizone Modeling Website http://www.bfrl.nist.gov/IAQanalysis. The user interface (or "Home Screen") is shown in Figure 1.

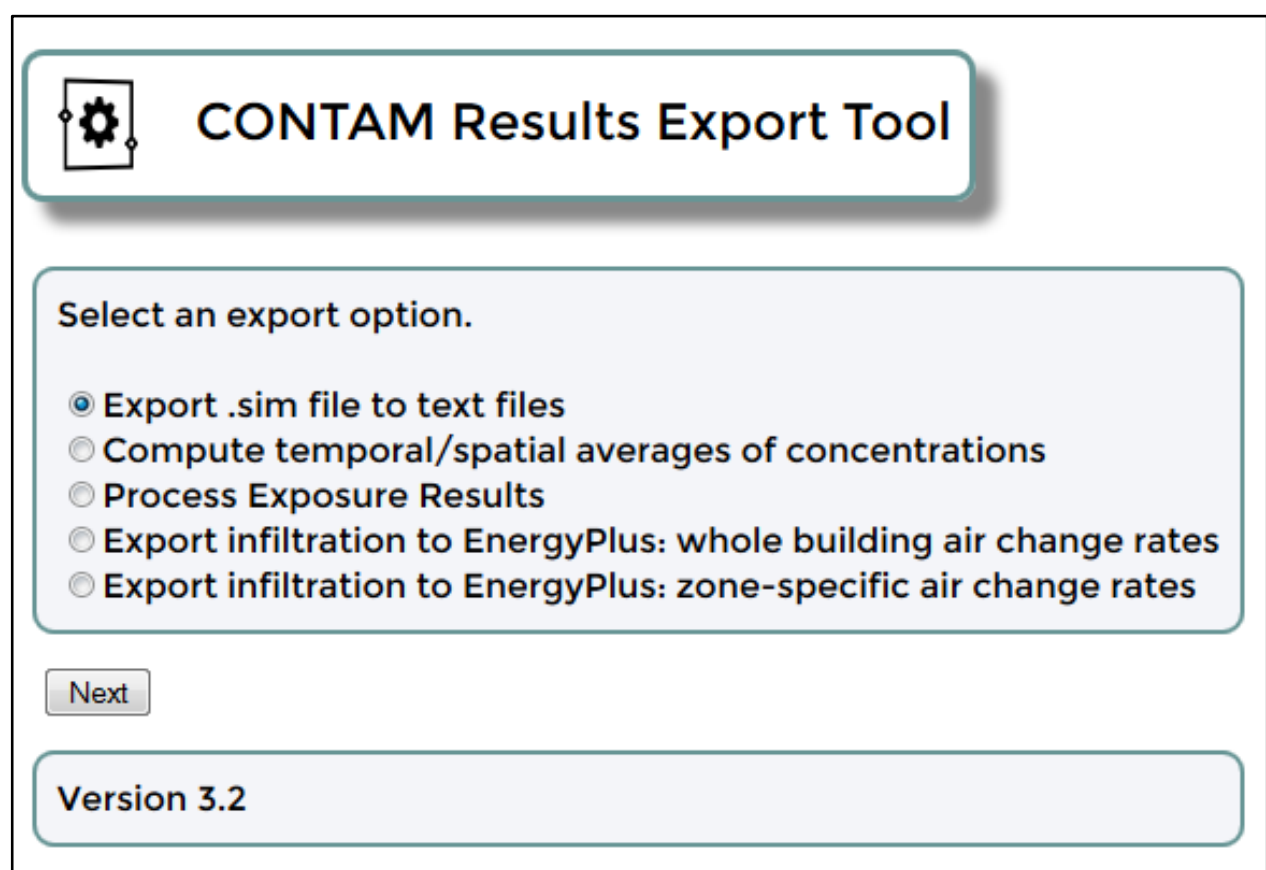

Figure 1. CONTAM Results Export Tool user interface (or "Home Screen") 


\section{Features}

The CONTAM Results Export Tool has five export options. Below is a short description of each, color-coded to match the organization of the document. Click on the feature name to go directly to the location in the document where it is described in more detail.

1. Export sim fille to text filles: Use this option to export building conditions (temperature, pressure differences, air density), ambient conditions (temperature, atmospheric pressure differences, wind speed, and wind direction), and contaminant concentrations (indoor, outdoor) to tab-delimited text files.

2. Compute temporal/spatial averages of concentrations: Use this option to export concentration results that are averaged temporally (over a user-defined period of time) or spatially (over a user-specified set of zones).

3. Process exposure results: Use this option to export exposure results organized by occupants or by contaminants.

4. Export infiltration to EnergyPlus: whole-building air change rates: Use this option to export whole-building infiltration rates for use in EnergyPlus.

5. Feature: Export infiltration to EnergyPlus: zone-specific air change rates: Use this option to export zone-specific infiltration rates for use in EnergyPlus. 


\section{Feature: Export sim filles to text fille}

Use this option to export indoor environmental conditions (zone temperature, zone gauge pressure, pressure differences across leakage paths, and air density), ambient environmental conditions (temperature, atmospheric pressure, wind speed, and wind direction), and contaminant concentrations (indoor, outdoor) to tab-delimited text files. Figure 2 presents a flow diagram of the steps involved in exercising this feature. The numbered/colored boxes refer to figure numbers throughout the step-by-step instructions that follow and that show screen shots of the Export Tool.

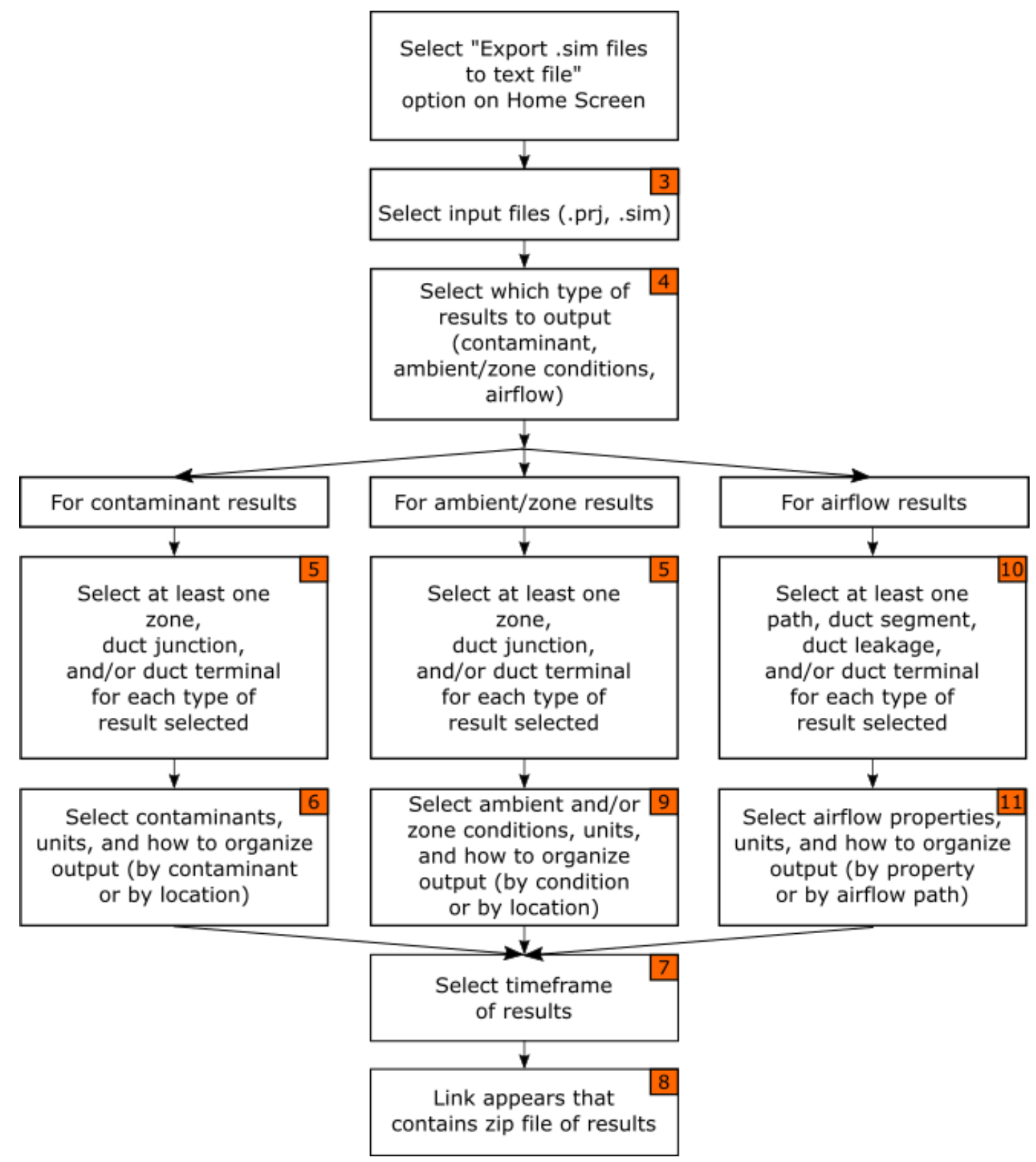

Figure 2. Flow diagram of the "Export .sim file to text files" feature 


\subsection{Step-by-step instructions for the "Export .sim files to text file" feature}

1. On the CONTAM Results Export Tool Home Screen (Figure 1), select the "Export .sim files to text files" radio button then click the "Next" button.

2. Select both a CONTAM project (.prj) and simulation results (.sim) file (Figure 3).

3. Choose from three types of results to output: Output Contaminant Concentration Results, Output Ambient and Zone Results, and Output Airflow Results (Figure 4).

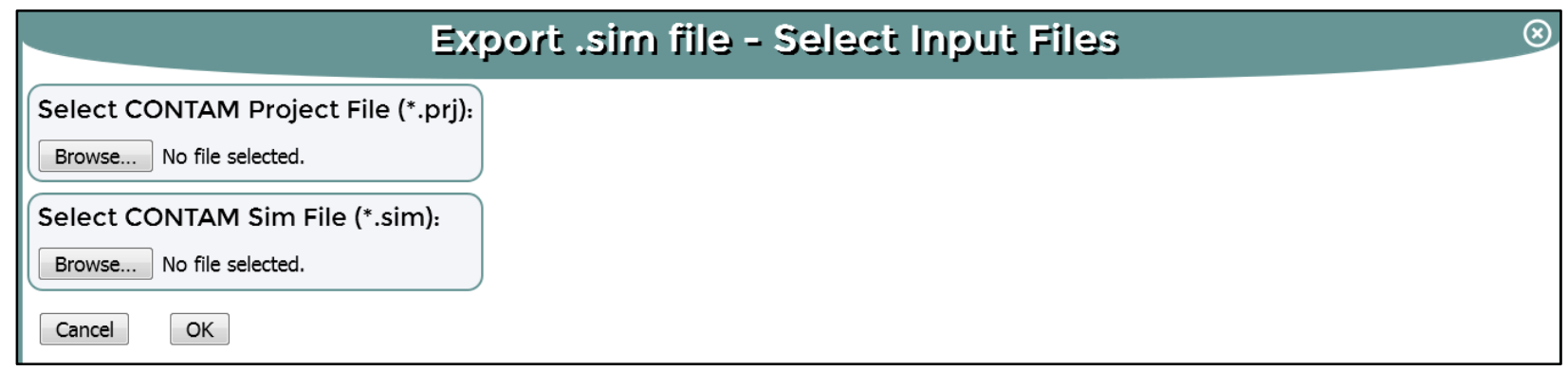

Figure 3. Selecting CONTAM project (.prj) and simulation results (.sim) files

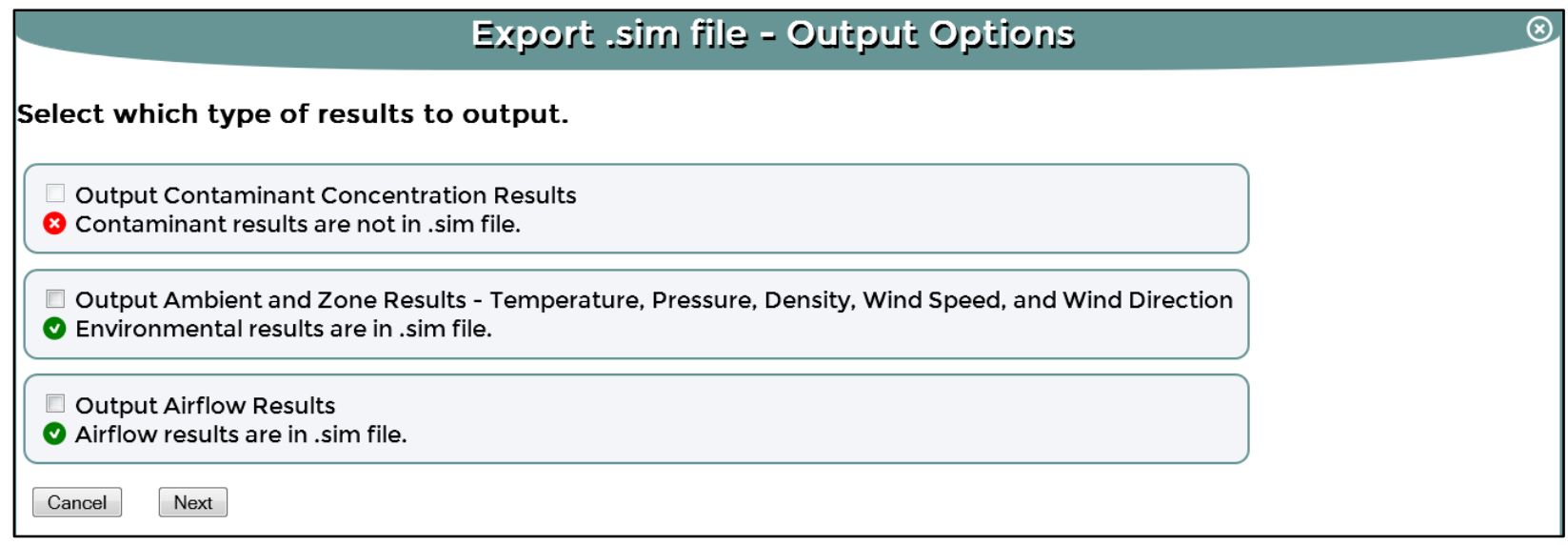

Figure 4. Three types of results within the "Export sim file to text files" feature Note: If the .sim file selected contains contaminant results (i.e., if contaminants were simulated), a green check mark will appear and a checkbox will be available to indicate that those results are available for exporting. If contaminants were not simulated, a red " $x$ " will appear and the checkbox will be unavailable to indicate that those results are unavailable for exporting. In general, the second two options ("ambient and zone" and "airflow") are always available.

\section{Output 1: Output Contaminant Concentration Results:}

4. Select the zones, duct junctions and/or duct terminals for which to output contaminant concentrations. You must select at least one item (Figure 5).

5. Select the options related to exporting contaminant concentrations (Figure 6):

a. Select which contaminants to output. You can select multiple contaminants using the Ctrl key. 
b. Select whether to output the ambient concentrations.

c. Select the output units of the contaminant concentrations from the dropdown menus. Different sets of units are provided for gaseous and particle contaminants.

d. Select how to organize the output. The first option, "Contaminants by Row/Zones, Junctions and Terminals by Column", means each row of the output file is for each contaminant and the columns contain the selected zones, ducts, and/or terminals. The second option is the opposite, where each row of the output file is for each selected zone, duct, and/or terminal, and the columns contain the selected contaminants.

6. Select the timeframe for which results are to be output (start date, start time, end date, end time). By default, the entire simulation period is selected (Figure 7).

7. Once you have clicked the "Finish" button, a link will be provided to a compressed zip file (Figure 8) that contains the "Node Contaminant Results" (.ncr) file, which contains the selected contaminant results. The name of the .ncr file defaults to the name of the project file (.prj) selected. Right-click on the link to either download the zip file to your computer, or to open the zip file and copy the extracted file(s) to your computer.

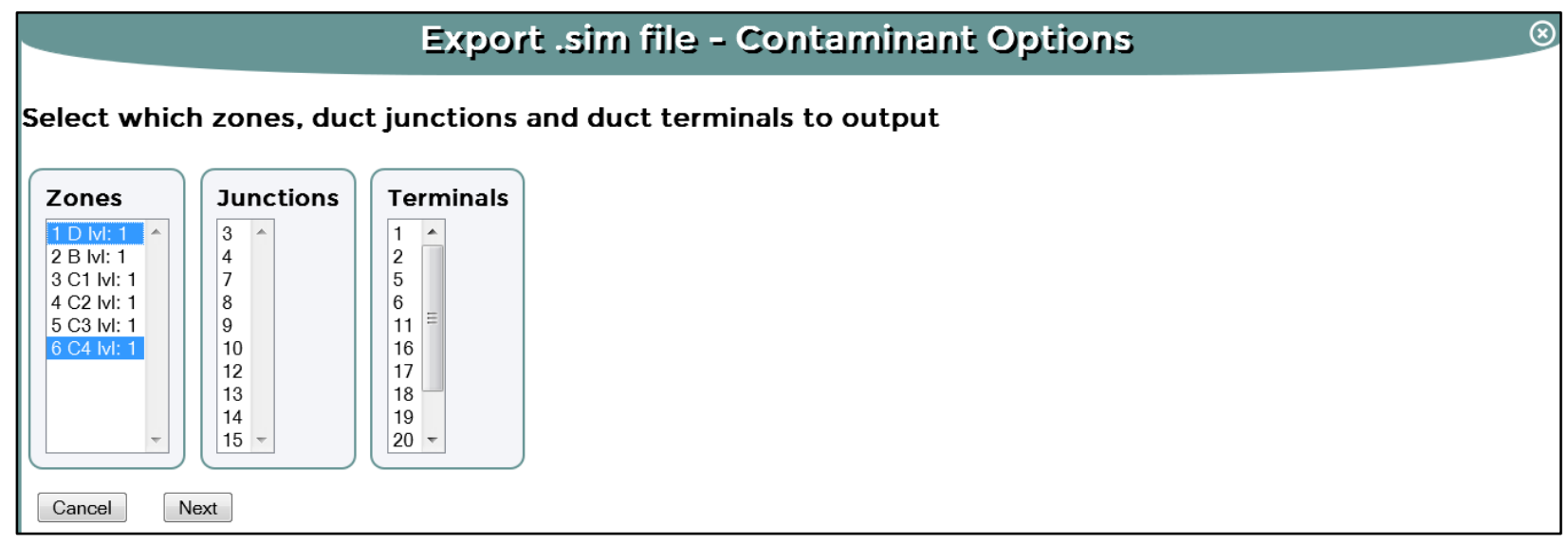

Figure 5. Output contaminant results (zone, junction, and/or terminal selection)

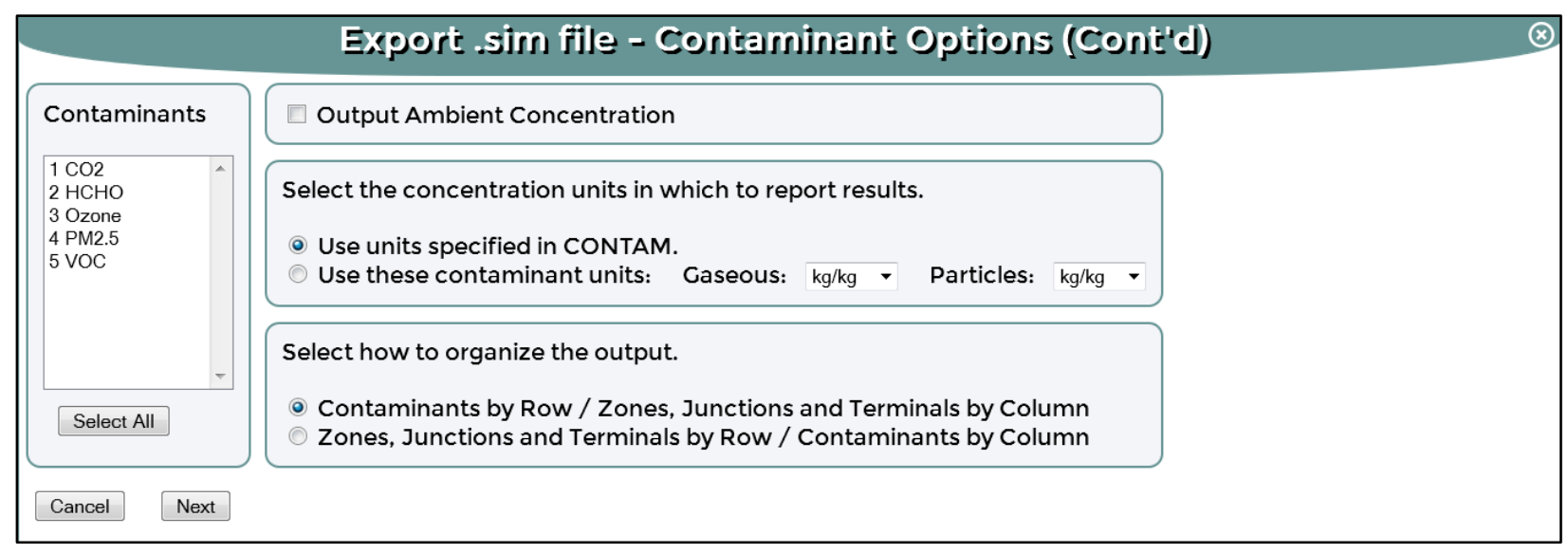

Figure 6. Output contaminant results (contaminants, units, and organization) 


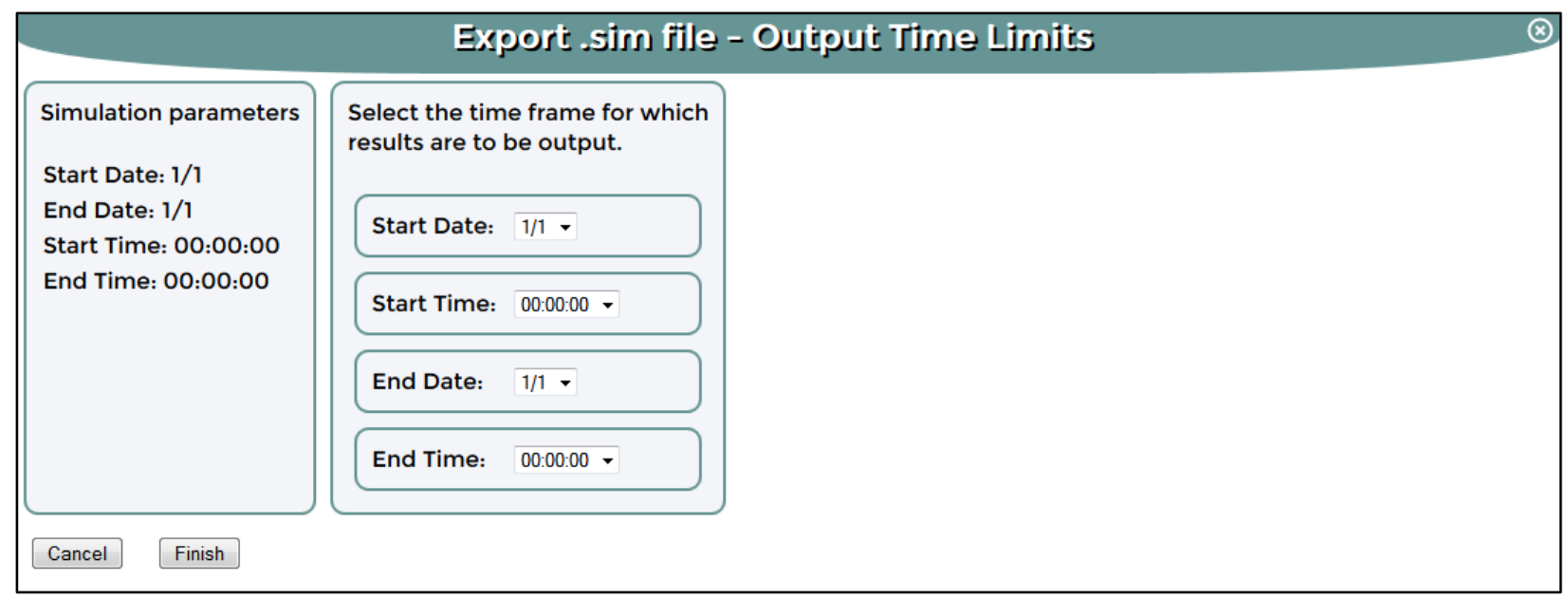

Figure 7. Output contaminant results (timeframe)

$$
\text { Export sim file - Save Files }
$$

Save Result Zip File

Figure 8. Output contaminant results (link containing zip files)

\section{Outpuit 2: Output Ambient and Zone Results:}

8. Select the zones, duct junctions and/or duct terminals for which ambient and/or zone results will be exported. You must select at least one item (Figure 5).

9. Select the options related to exporting ambient and/or zone conditions (Figure 9):

a. Select the building conditions (temperature, zone gauge pressure, density) to output for each of the zones, duct junctions and/or terminals.

b. If desired, select the ambient conditions to output (temperature, atmospheric pressure, wind speed, wind direction).

c. Select the units desired for each output. Units of wind direction are degrees.

d. Select how to organize the output. The first option, "Conditions by Row/Zones, Junctions and Terminals by Column", means each row of the output file is for each selected ambient/zone condition and the columns contain the selected zones, ducts, and/or terminals. The second option is the opposite, where each row of the output file is for each selected zone, duct, and/or terminal, and the columns contain the selected ambient/zone conditions.

10. Select the timeframe for which results are to be output (start date, start time, end date, end time). By default, the entire simulation period is selected (Figure 7).

11. Once you have clicked the "Finish" button, a link will be provided to a compressed zip file (Figure 8) that contains the Node Flow Results" (.nfr) file, which contains the selected ambient/zone conditions. The name of the .nfr file defaults to the name of the project file (.prj) selected. Right-click on the link to either download the zip file to your computer, or to open the zip file and copy the extracted file(s) to your computer. 


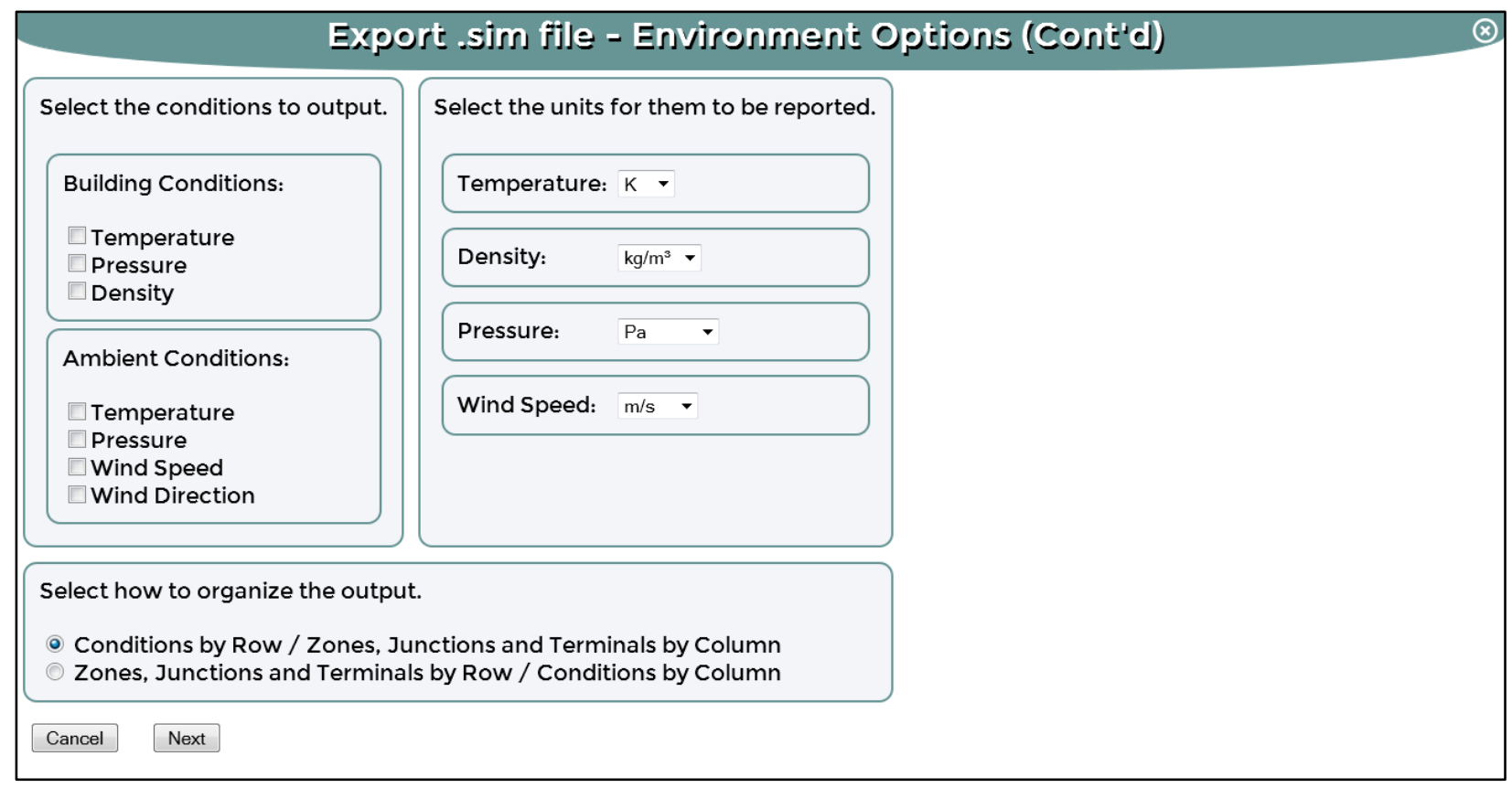

Figure 9. Output ambient and zone results

\section{Output 3: Output Airflow Results:}

12. Select the paths, duct segments, duct leakages and/or duct terminals to output airflow results for. "Paths" are airflow elements such as doors, windows, and wall leakages. You must select at least one item (Figure 10).

13. Select the options related to exporting airflow results (Figure 11):

a. Select the properties for each path, duct segment, duct leakage, and/or duct terminal to output, i.e., pressure difference and flow ("Flow 1" and/or "Flow 2") across the airflow path, duct segment, duct leakage or duct terminal. There will only be a value for "Flow 2" for airflow paths that utilize two-way flow elements (Dols and Polidoro 2015). The signs of the pressure differences and flow values depend on the direction chosen as positive in the CONTAM model for each path, duct terminal and/or duct segment. Duct leakage does not have assigned positive directions.

b. Select the units desired for each output.

c. Select how to organize the output. The first option, "Airflow Properties by Row/ Airflow Properties by Column", means each row of the output file is for the selected airflow properties and the columns contain the selected airflow paths chosen. The second option is the opposite, where each row of the output file is for each selected airflow path, and the columns contain the selected airflow properties.

14. Select the timeframe for which results are to be output (start date, start time, end date, end time). By default, the entire simulation period is selected (Figure 7). 
15. Once you have clicked the "Finish" button, a link will be provided to a compressed zip file (Figure 8) that contains the "Link Flow Results" (.1fr) file, which contains the selected airflow results. The name of the .lfr file defaults to the name of the project selected. Rightclick on the link to either download the zip file to your computer, or to open the zip file and copy the extracted file(s) to your computer.

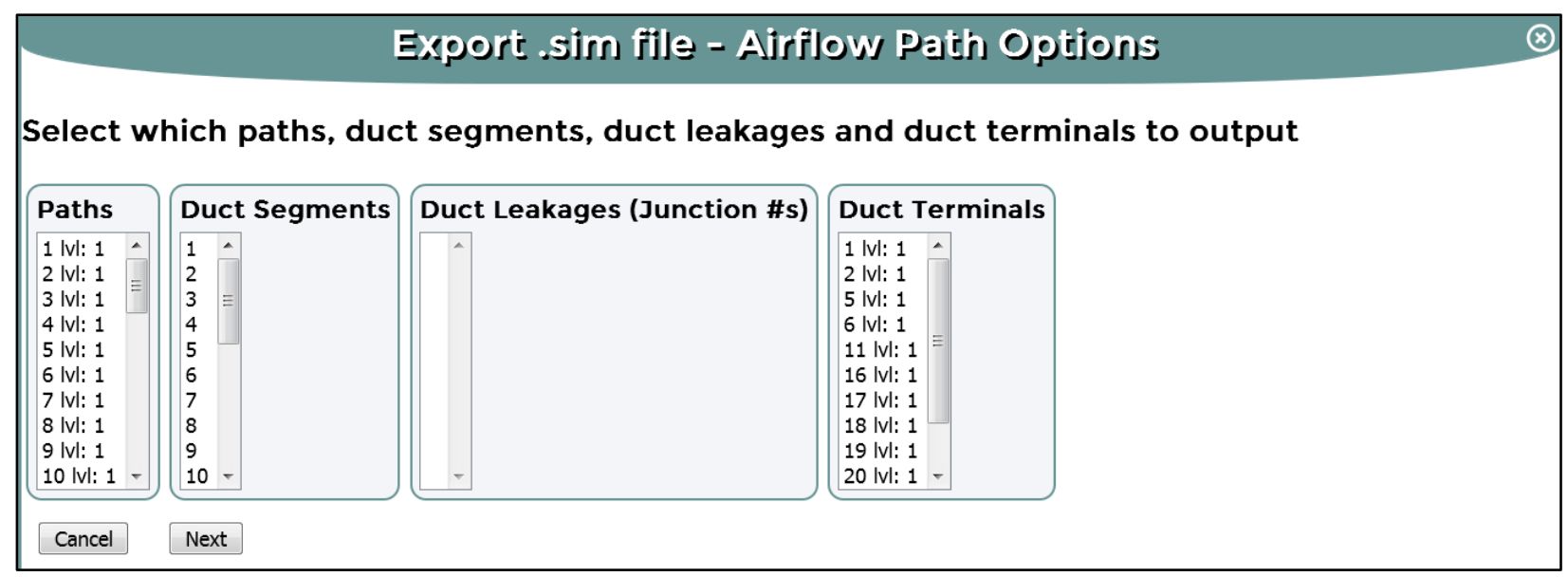

Figure 10. Output airflow results (path, duct segments, duct leakages, and/or duct terminal selection)

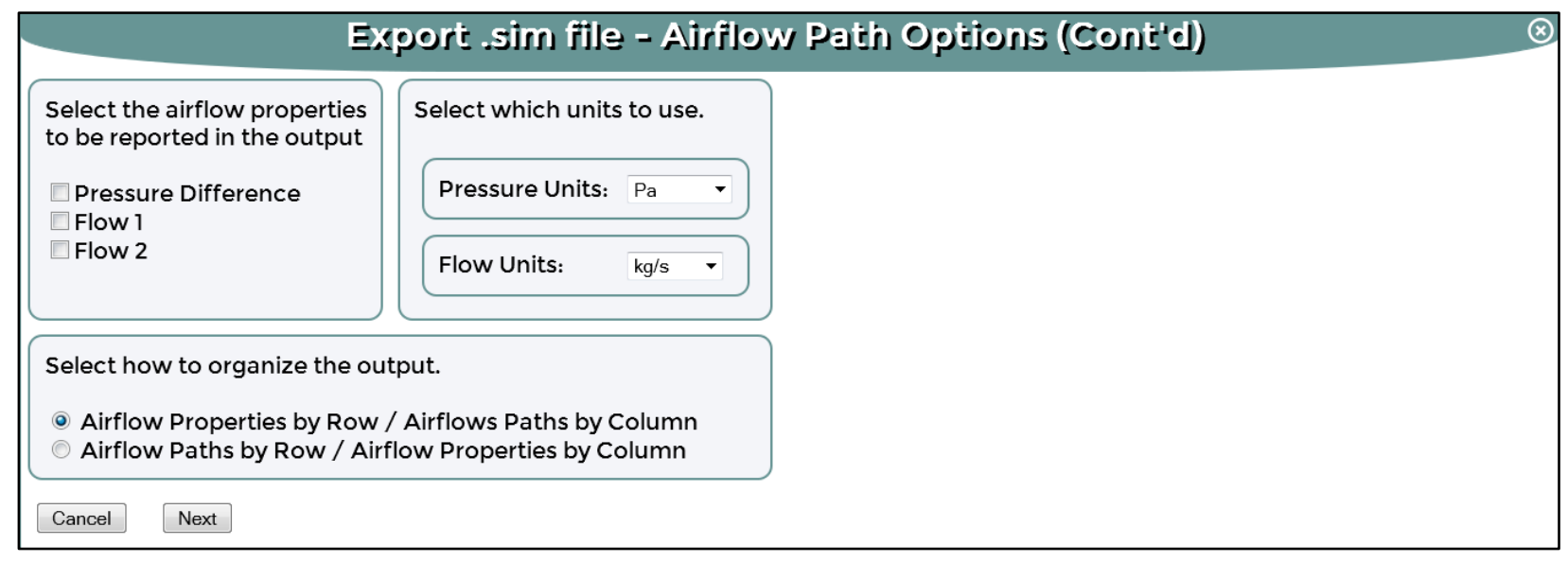

Figure 11. Output airflow results (airflow properties, units, and organization) 


\section{Feature: Compute temporal/spatial averages of concentrations}

Use this option to export contaminant concentrations directly, averaged over a user-selected period of time, or averaged over a user-selected set of zones. Averages can also be computed for duct junctions and/or duct terminals. Zone-averaged concentrations will be volume-weighted. Figure 12 presents a flow diagram of the steps involved in exercising this feature. The numbered/colored boxes refer to figure numbers throughout the step-by-step instructions that follow and that show screen shots of the Export Tool. The orange boxes refer to figures presented in the previous section "Feature: Export sim files to text file". The green boxes refer to figures in this section.



Figure 12. Flow diagram of the "Compute temporal/spatial averages of concentrations" feature 


\subsection{Step-by-step instructions for the "Compute temporal/spatial averages of concentrations" feature}

1. On the CONTAM Results Export Tool Home Screen (Figure 1), select the "Compute temporal/spatial averages of concentrations" radio button then click the "Next" button.

2. Select both a CONTAM project (.prj) file and simulation results (.sim) file (Figure 3).

3. Select averaging option(s) to perform - temporal, spatial, or both (Figure 13).

4. Select the zones, duct junctions and/or duct terminals for which averages are desired. This selection will be done twice if both temporal and spatial averages are selected in the previous step. You must select at least one item (Figure 5).

5. Select the contaminants to average, whether to output the ambient concentrations, the output units of the contaminant concentrations, and how to organize the output (Figure 6).

6. Select the total timeframe for which to output temporal and/or spatial average concentrations (Figure 14). By default, the whole simulation period will be selected. Next, select the averaging sub-interval over which the temporal averages are to be calculated. This is done by selecting the number of time steps to average. The minimum number is two. There is no maximum, however, if the number of time steps selected is greater than the number of time steps in the simulation, then the temporal average is calculated using the number of time steps in the simulation.

7. Once you have clicked the "Finish" button, a link will be provided to a compressed zip file which contains the selected contaminant results (Figure 8). Spatially averaged result files will have a .savg file extension, and time-averaged result files will have a tavg file extension. The names of the .savg and .tavg files default to the name of the project selected. Right-click on the link to either download the zip file to your computer, or open the zip file and copy the extracted file(s) to your computer.

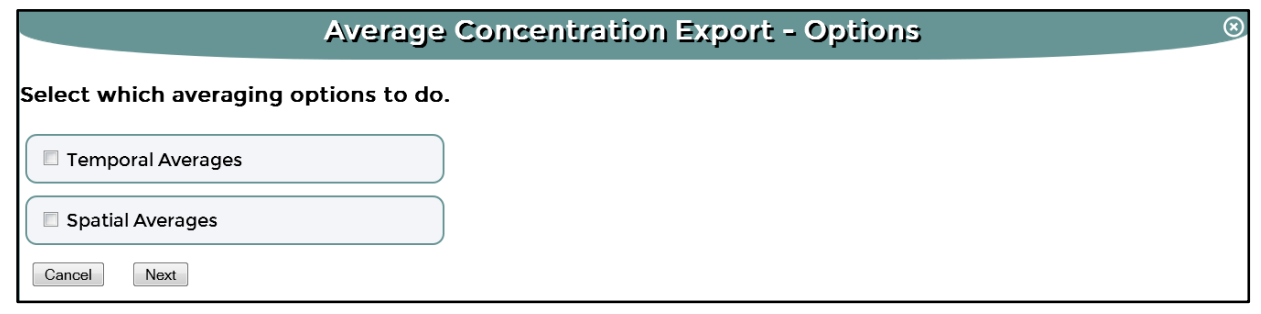

Figure 13. Compute temporal/spatial averages of concentrations (averaging options)



Figure 14. Compute temporal/spatial averages of concentrations (timeframe) 


\section{Feature: Process exposure results}

This feature will only be available if the option to export an occupant exposure file (.ebw) was set via the ContamW simulation output parameters. The .ebw file contains the results for each occupant and contaminant simulated in the .prj file (Dols and Polidoro 2015). This feature will create a separate file for each occupant or for each contaminant simulated. The results can be exported for a subset of the simulation time period. Figure 15 presents a flow diagram of the steps involved in exercising this feature. The numbered/colored boxes refer to figure numbers throughout the step-by-step instructions that follow and that show screen shots of the Export Tool. The orange box refers to a figure presented in the previous section "Feature: Export .sim files to text file". The blue boxes refer to figures in this section.

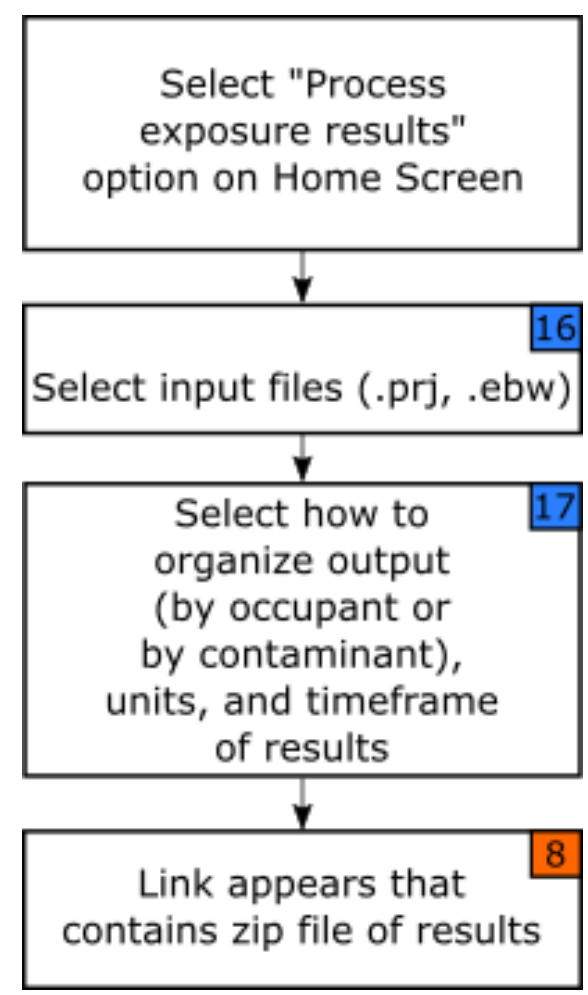

Figure 15. Flow diagram of the "Process exposure results" feature 


\subsection{Step-by-step instructions for the "Process exposure results" feature}

1. On the CONTAM Results Export Tool Home Screen (Figure 1), select the "Process exposure results" radio button then click the "Next" button.

2. Select both a CONTAM project (.prj) file and an exposure box-whisker (.ebw) file (Figure 16).

3. Select the options related to exporting exposure results (Figure 17):

a. Select the output format, either one file for each occupant (and its exposure to each contaminant), or one file for each contaminant (and exposure of all occupants to it).

b. Select the output units of the exposure concentrations from the dropdown menus. Different sets of units are provided for gaseous and particle contaminants.

c. Select the time period to output (start date, start time, end date, end time). By default, the whole simulation period will be selected.

4. Once you have clicked the "Finish" button, a link will be provided to a compressed zip file (Figure 8) that contains the "Personal Exposure File" (.pxf) file, which contains the selected exposure results. The names of the .pxf files default to the name of the project selected followed by an underscore and either the occupant exposure object number or the contaminant name. Right-click on the link to either download the zip file to your computer, or to open the zip file and copy extracted the file(s) to your computer.



Figure 16. Selecting CONTAM project (.prj) and exposure box-whisker file (.ebw) files

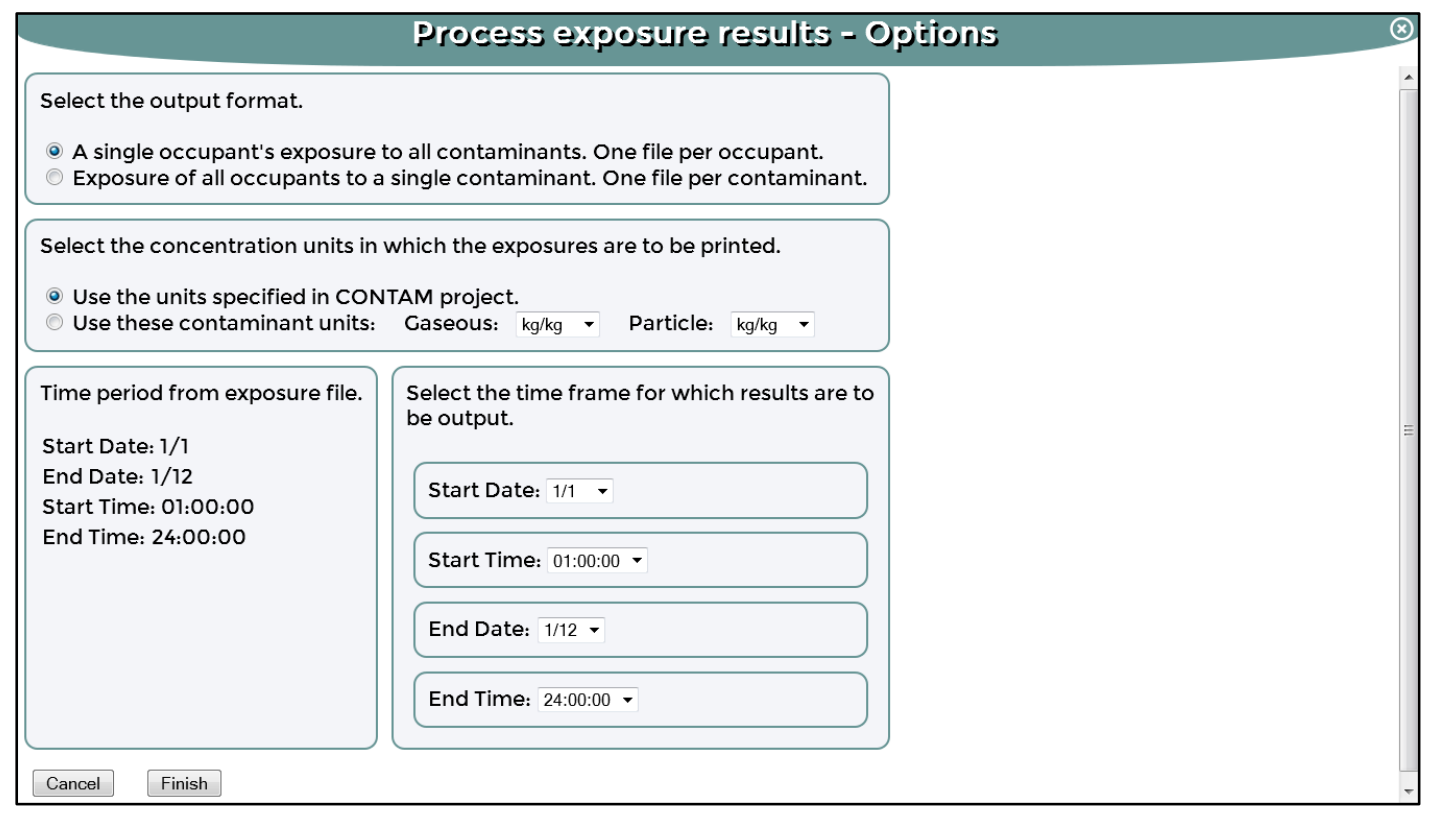

Figure 17. Processing exposure results (organization, units, and timeframe) 


\section{Feature: Export infiltration to EnergyPlus: whole-building air change rates}

\subsection{Background: Modeling infiltration in EnergyPlus}

EnergyPlus is a building energy simulation software developed by the U. S. Department of Energy (DOE). It has several infiltration models, including ZoneInfiltration:DesignFlowRate (DOE 2013). This model uses the following empirical equation to calculate infiltration:

$$
\text { Infiltration }=I_{\text {design }} \bullet F_{\text {schedule }}\left[A+B|\Delta T|+C \cdot W_{\mathrm{s}}+D \bullet W_{\mathrm{s}}^{2}\right]
$$

where:

$I_{\text {design }}$ is defined by EnergyPlus as the "maximum amount of infiltration expected at design conditions". According to the documentation, EnergyPlus provides several options to input this design flow rate $\left(\mathrm{h}^{-1}, \mathrm{~m}^{3} / \mathrm{s} \bullet \mathrm{m}^{2}, \mathrm{~m}^{3} / \mathrm{s}\right)$, which is ultimately converted to mass flow using the outdoor air density at each time step.

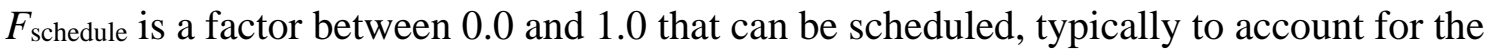
impacts of the heating, ventilating, and air conditioning (HVAC) system operation on infiltration.

$|\Delta T|$ is the absolute indoor-outdoor temperature difference in ${ }^{\circ} \mathrm{C}$.

$W_{\mathrm{s}}$ is the wind speed in $\mathrm{m} / \mathrm{s}$.

$A, B, C$, and $D$ are constants, for which values are suggested in the EnergyPlus user manual (DOE 2013). However, those values are based on studies in low-rise residential buildings and do not generally apply to taller buildings and mechanically ventilated buildings. See $\mathrm{Ng}$ et al. (2014) for an alternative approach to generating $A, B, C$, and $D$ for improved infiltration modeling using this equation. Given the challenges in determining valid coefficients for a given building, a common strategy used in EnergyPlus for incorporating infiltration is to assume fixed infiltration rates, sometimes using a different constant value depending on whether the HVAC system is on or off. However, this strategy does not reflect known dependencies of infiltration on outdoor weather and the complexities of HVAC system operation.

Multizone airflow modeling is the accepted approach to calculate infiltration based on the fundamental airflow physics (Walton 1989). The AIRFLOW NETWORK model in EnergyPlus is based on an early version of CONTAM with restrictions on the type of analyses that it can perform, including only being able to include the effects of a single heating, ventilating, and air conditioning (HVAC) system on infiltration (DOE 2013). The full CONTAM model is not restricted this way, so in order to incorporate more physically-reasonable and wholebuilding/system infiltration calculations into EnergyPlus simulations, the CONTAM Results Export Tool provides users two ways to incorporate CONTAM infiltration rates into an EnergyPlus model. One feature exports whole building air change rates, and the other exports 
zone-specific air change rates. Users of these features need to be familiar with how to perform CONTAM simulations (Dols and Polidoro 2015) and how to modify EnergyPlus IDF (input data format) files using either the EnergyPlus IDF Editor program or a text editor.

The feature that exports whole-building air change rates for use in EnergyPlus creates a schedule of $I_{\text {design }}$ values that will be used by EnergyPlus to calculate infiltration rates based on Equation (1), with $A=1$ and $B=C=D=0$. Using the whole building's infiltration rates calculated by CONTAM, this feature normalizes the infiltration rates by the building's exterior above-grade surface area using the following equation:

$$
I_{\text {design }}\left(\mathrm{m}^{3} / \mathrm{s} \bullet \mathrm{m}^{2} \text { for "whole-building” feature }\right)=N \cdot V /\left(3600 A_{s}\right)
$$

where:

$N$ is the whole building air change rate $\left(\mathrm{h}^{-1}\right)$ due only to exterior flow paths calculated by CONTAM.

$V$ is the building volume $\left(\mathrm{m}^{3}\right)$ calculated by CONTAM.

$A_{s}$ is the building exterior surface area $\left(\mathrm{m}^{2}\right)$, i.e., the gross surface areas of the abovegrade walls and the roof, entered by the user.

For this feature, the same $I_{\text {design }}\left(\mathrm{m}^{3} / \mathrm{s} \bullet \mathrm{m}^{2}\right)$ will be applied to each zone with exterior surfaces (or "exterior zones") in the EnergyPlus model, thereby distributing the infiltration uniformly over the entire building envelope. Since $I_{\text {design }}$ is normalized by exterior surface area, zones with more exterior surfaces (wall, roof) will have more infiltration than other zones, and interior zones will receive no infiltration air in the EnergyPlus model.

\subsection{Limitations and Other Considerations}

This feature assumes that envelope leakage is uniformly distributed around the building envelope. It neglects the effects of wind direction on infiltration and its distribution among zones. If a user requires a more detailed information on infiltration, then the zone-specific feature described in the next section is available. The following constraints are also placed on the CONTAM simulation:

a. Both the CONTAM and EnergyPlus simulations start on January 1, 00:00:00 and end on December 31, 24:00:00 for the whole-building export option. For the zone-specific export option, it must start at the beginning of a day and end at the end of a day.

b. The number of time steps in the CONTAM simulation must be at least 8760 . The smallest time step allowed in EnergyPlus is one minute. Ideally, the time step in the CONTAM and EnergyPlus simulations should match.

c. Do not simulate Daylight Savings in CONTAM or in the EnergyPlus simulation.

d. The HVAC system configuration and operating schedules in the CONTAM model should match the configuration in the EnergyPlus model. This is important so that the infiltration calculated by CONTAM, which is then input into EnergyPlus, will account for the HVAC system effects on infiltration. If the HVAC configurations 
between CONTAM and EnergyPlus do not match, such as when variable volume air (VAV) systems or economizers are modeled, EnergyPlus simulations can still be performed using the output files from this tool.

e. If there are intake fans in the building envelope that are modeled in CONTAM as flow paths, then the airflow through those fans will be included in the infiltration rate by this tool. To prevent this from happening, model intake fans using a duct instead of a flow path.

It should be noted that this feature was developed for use with EnergyPlus v8.2. For earlier versions of EnergyPlus, see the EnergyPlus documentation for the format of the ScheduleTypeLimits, Schedule:File, and ZoneInfiltration:DesignFlowRate objects created by this tool that may require editing before running an EnergyPlus simulation.

\subsection{What's created by the feature}

This feature will only be available if the option to export detailed building air change rates (.ach) was set via the ContamW simulation output parameters. The ach file contains the wholebuilding air change rates simulated in the .prj file (Dols and Polidoro 2015). This feature will create a partial input data file (partial .idf) file and a comma-separated values (.csv) file.

The partial .idf file contains the following EnergyPlus objects (Figure 18):

- ScheduleTypeLimits - This is a generic limits object that is required by the Schedule:File object. It assigns the values in the Schedule:File object as dimensionless.

- Schedule:File - This object calls the .csv file (described below) that is created by this feature and contains the $I_{\text {design }}$ Values.

- ZoneInfiltration:DesignFlowRate - This object calls the Schedule:File object for the values of $I_{\text {design }}$ calculated using CONTAM infiltration rates. This object applies the $I_{\text {design }}$ values to any or all of the zones in the EnergyPlus file. The user can apply $I_{\text {design }}$ values to multiple zones by copying and pasting a separate ZoneInfiltration:DesignFlowRate object for each zone and entering a unique Zone Name for the "Zone or ZoneList Name" field for each ZoneInfiltration:DesignFlowRate object. The user can also apply $I_{\text {design }}$ values to a group of zones by creating a ZoneList and entering the name of the ZoneList object for the "Zone or ZoneList Name" field of the ZoneInfiltration:DesignFlowRate object (recommended since it reduces error in transferring parameter values from the partial idf file to the EnergyPlus project file). It may also be beneficial to the user to create the ZoneList containing only the exterior zones, though if it contains the names of interior zones, the ZoneInfiltration:DesignFlowRate object created by this feature will only calculate infiltration for zones with above-grade exterior surfaces (walls and roof). 


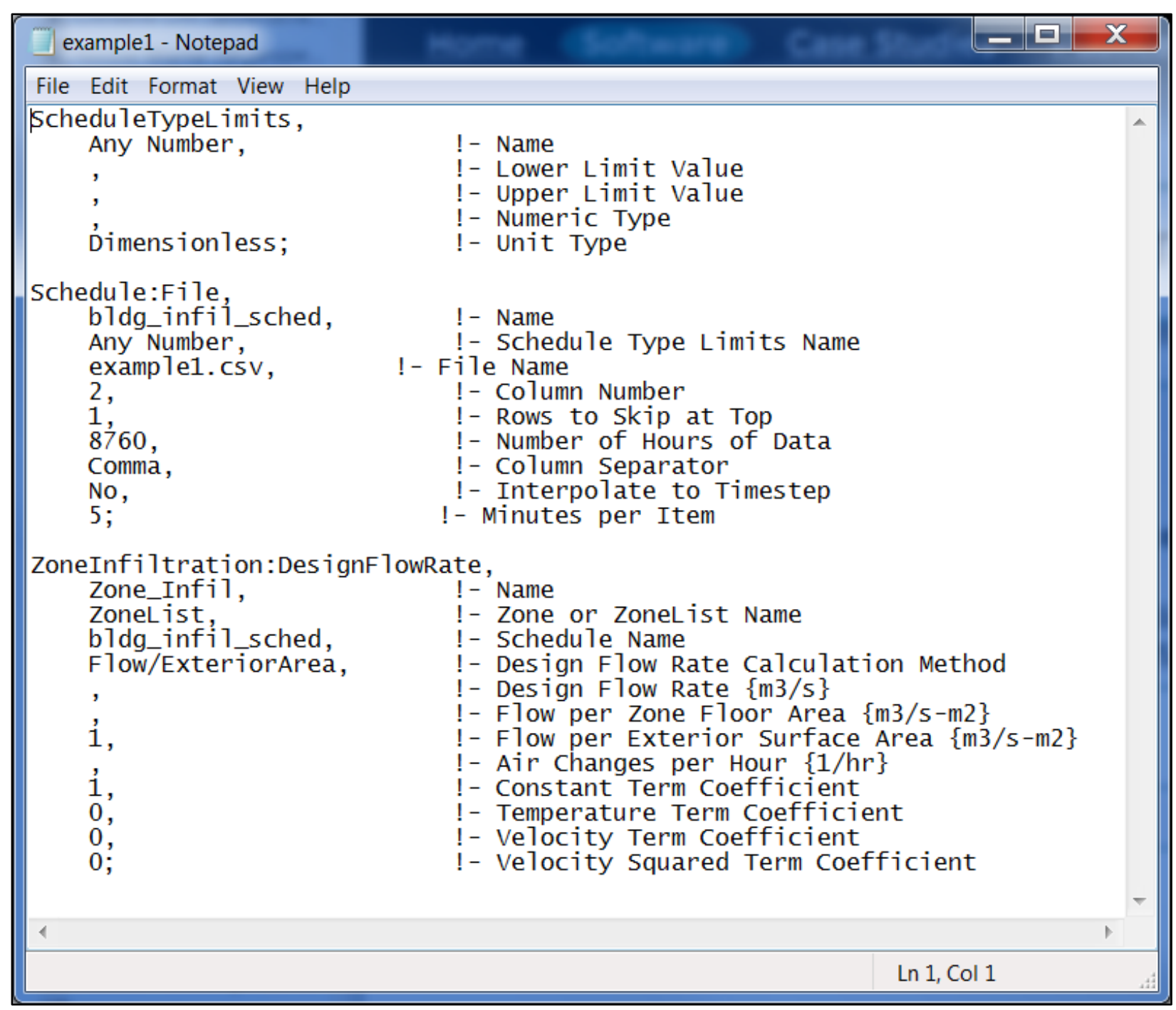

Figure 18. Partial .idf file created by "Export infiltration to EnergyPlus: whole-building air change rates" feature

The .csv file is a schedule of $I_{\text {design }}$ values calculated using the CONTAM infiltration rates (Figure 19). These values are called during the EnergyPlus simulation by the Schedule:File object. The .csv file needs to be located in the same directory as the EnergyPlus project file. Step-by-step instructions on how to use these files in an EnergyPlus project will be given next. 


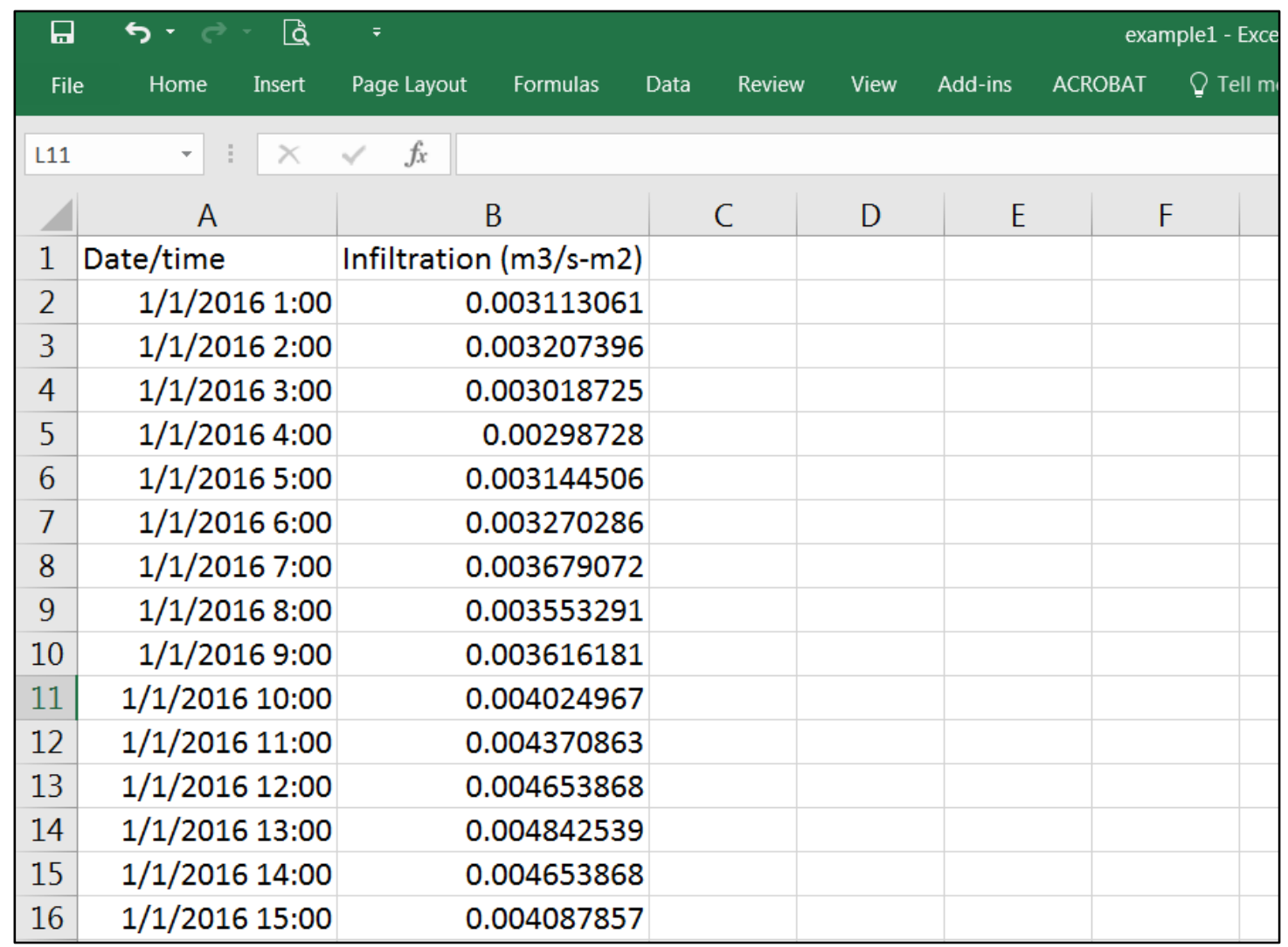

Figure 19. .csv file created by "Export infiltration to EnergyPlus: whole-building air change rates" feature

\subsection{Using the feature}

Figure 20 presents a flow diagram of the steps involved in exercising this feature. The numbered/colored boxes refer to figure numbers throughout the step-by-step instructions that follow and that show screen shots of the Export Tool.

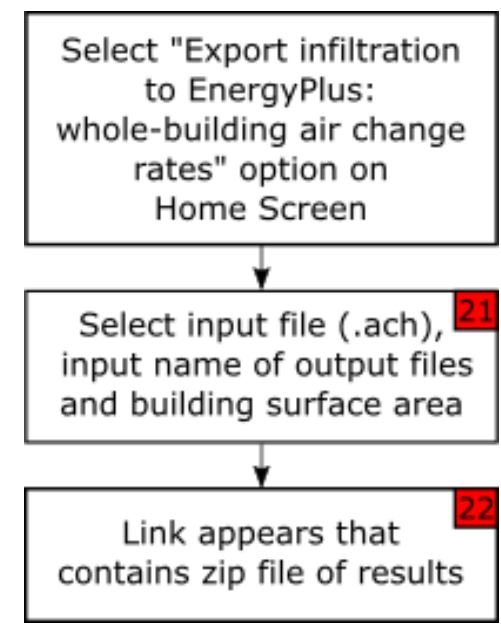

Figure 20. Flow diagram of the "Export infiltration to EnergyPlus: whole-building air change rates" feature 


\subsection{Step-by-step instructions for the "Export infiltration to EnergyPlus: whole-building air change rates" feature}

1. On the CONTAM Results Export Tool Home Screen (Figure 1), select the "Export infiltration to EnergyPlus: whole-building air change rates" radio button then click the "Next" button.

2. Complete the options related to exporting whole-building air change rates (Figure 21):

a. Select a CONTAM air changes per hour (.ach) file.

b. If you do not want the filenames given to the partial idf and .csv files created by the tool to be the same as that of the .ach file chosen, change it here. Commas are not allowed in the filename.

c. Enter the total above-grade gross areas of exterior walls and the roof area of the building modeled. The user can obtain this value from the results of an EnergyPlus simulation under the InputVerificationandResultsSummary section of Output:Table:SummaryReports.

d. The defaults units are $\mathrm{m}^{2}$, which is required by the EnergyPlus simulation. If you would like the air change rates to be in another unit, select from the dropdown menu.

3. Once you have clicked the "OK" button, a link will be provided to a compressed zip file (Figure 22) that contains the partial .idf and .csv files, which contains the whole-building air change rates normalized by above-grade gross exterior area and EnergyPlus input objects. The use of these files in an EnergyPlus simulation will be described next. Right-click on the link to either download the zip file to your computer, or to open the zip file and copy extracted the file(s) to your computer.

\begin{tabular}{|c|c|c|}
\hline \multicolumn{3}{|c|}{ EnergyPlus Whole Building ACH Export - Options } \\
\hline Select a CONTAM ACH File (*.ach): & \begin{tabular}{|l|l} 
Browse... No file selected. \\
\end{tabular} & \\
\hline Enter a name for the output files: & file & \\
\hline IDF filename: & file.idf & \\
\hline CSV Schedule filename: & file.csv & \\
\hline Building Surface Area & 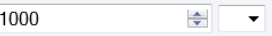 & \\
\hline Cancel $\mathrm{OK}$ & & \\
\hline
\end{tabular}

Figure 21. Options for the "Export infiltration to EnergyPlus: whole-building air change rates" feature

$$
\text { Energyplus Whole Building ACH Export - Save Files }
$$

Save zip file with $\mathrm{ACH}$ export files

Figure 22. Exporting whole-building air change rates (link containing zip files) 


\subsection{What to do with the .csv and partial .idf files}

Figure 23 presents a flow diagram of the steps to utilize the files generated by the feature in an EnergyPlus simulation. The numbered/colored boxes refer to figure numbers throughout the step-by-step instructions that follow and that show screen shots of the text editor and EnergyPlus IDF Editor program.

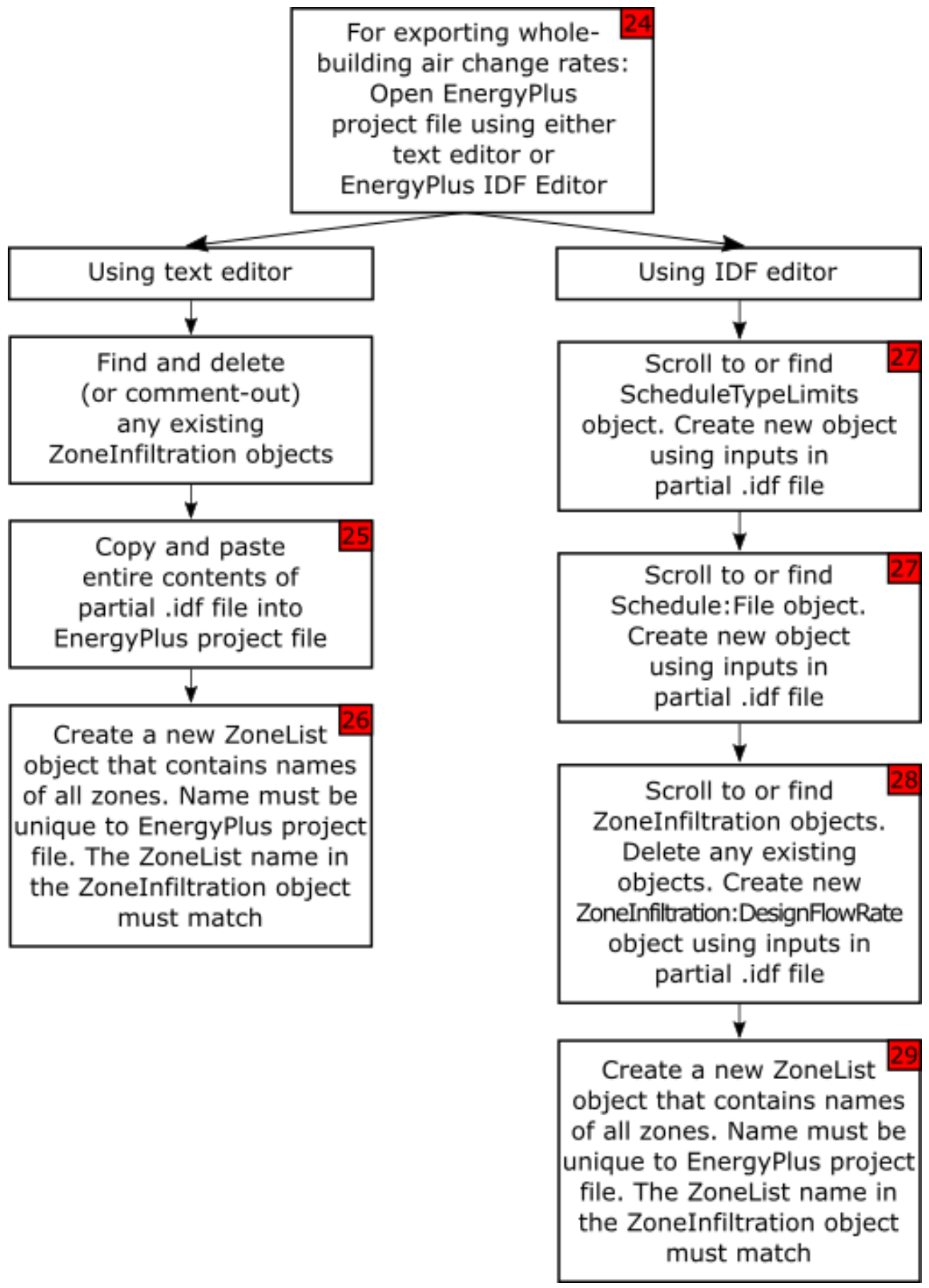

Figure 23. Flow diagram of what to do with the files generated by the "Export infiltration to EnergyPlus: whole-building air change rates" feature 
1. Open the EnergyPlus project file, into which you want to import CONTAM infiltration rates, with either a text editor or the EnergyPlus IDF Editor program (Figure 24).

2. If using a text editor:

a. Use the Find function $(\mathrm{Ctrl}+\mathrm{F})$ to locate any existing ZoneInfiltration objects. Delete any existing ZoneInfiltration objects or make them comment lines by adding an exclamation point (!) at the beginning of each line.

b. Copy and paste the entire contents of the partial idf created by this feature into the EnergyPlus simulation file. It does not matter where, however do not break-up any existing EnergyPlus objects by pasting the text from the partial idf file below an input that ends in a semicolon (Figure 25). If you would like similar objects to be grouped together, you can open up the updated EnergyPlus simulation file in the EnergyPlus IDF Editor program. When you use the "Save as" function, the objects in the EnergyPlus simulation file will re-order the objects and group similar objects together.

c. The feature creates one ZoneInfiltration:DesignFlowRate object that applies to the Zone or ZoneList Name "ZoneList" (Figure 18). Thus, you need to create a Zone List object in the EnergyPlus project file that contains the names of all the zones in the EnergyPlus project. An example is shown in Figure 26. You can find a list of all the zones in the EnergyPlus project file in the Zone object.

Since the ZoneInfiltration:DesignFlowRate object created by this feature specifies that only zones with above-grade exterior surface areas will receive infiltration, the ZoneList can contain the names of interior zones. If there are existing ZoneList objects in EnergyPlus project file, be sure that none are named "ZoneList". If any are, change the name of the new ZoneList object to anything you choose. Be sure to also copy this name to the ZoneInfiltration:DesignFlowRate object under Zone or ZoneList Name.

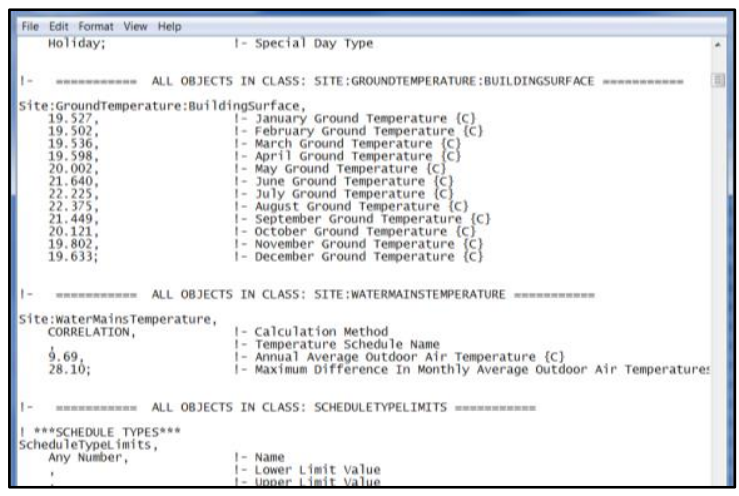

(a)



(b)

Figure 24. Open (a) text editor or (b) EnergyPlus IDF Editor program 


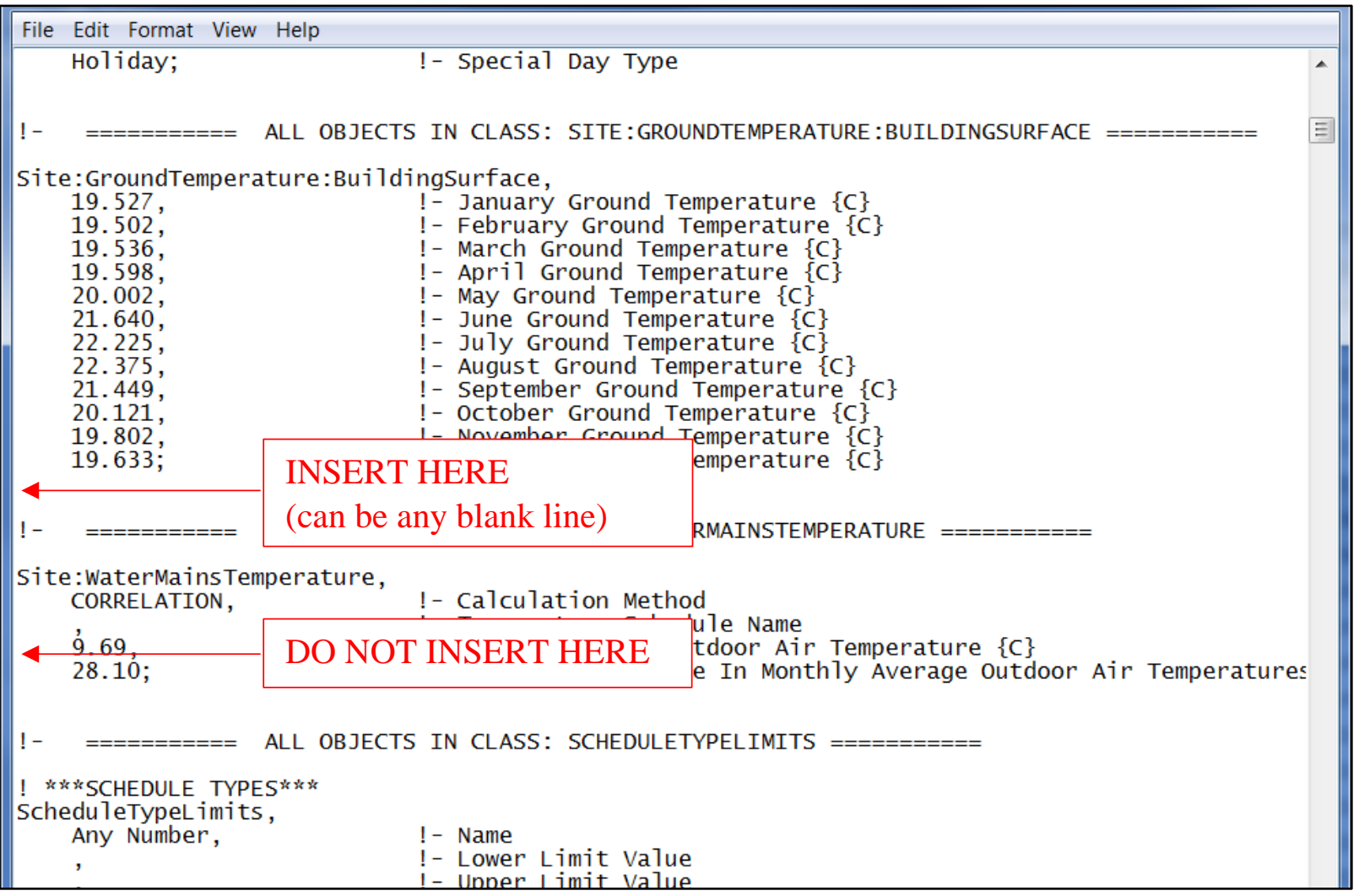

Figure 25. Where to insert the contents of the partial .idf file in EnergyPlus project file

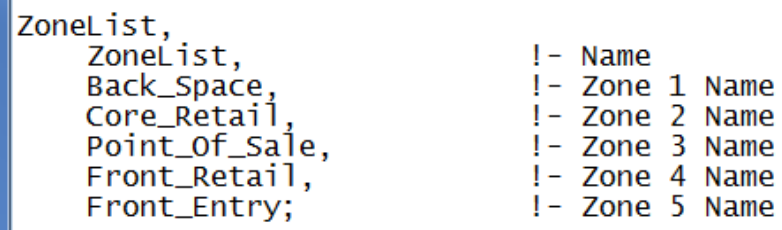

Figure 26. Example of ZoneList object inputs

3. If using the EnergyPlus IDF Editor program:

a. Scroll or use the Find function $(\mathrm{Ctrl}+\mathrm{F})$ to go to the ScheduleTypeLimits object (Figure 27). Press the "New Obj" button. In the new ScheduleTypeLimits object, input the following (the inputs are also in the partial .idf file):

i. Name: Any Number

ii. Unit Type: Dimensionless

b. Scroll or use the Find function (Ctrl+F) to go to the Schedule:File object (Figure 27). Press the "New Obj" button. In the new Schedule:File object, input the following (the inputs are also in the partial idf file):

i. Name: bldg_infil_sched

ii. Schedule Type Limits Name: Any Number

iii. File Name: copy the name of the .csv file created by this feature. For example, Figure 18 shows that filename is "example1.csv". 
iv. Column Number: 2

v. Rows to Skip at Top: 1

vi. Number of Hours of Data: 8760

vii. Column Separator: Comma

viii. Interpolate to Timestep: No

ix. Minutes per Item: 60

c. Scroll or use the Find function $(\mathrm{Ctrl}+\mathrm{F})$ to go to the ZoneInfiltration:DesignFlowRate object (Figure 28). Delete any existing ZoneInfiltration:DesignFlowRate objects. Press the "New Obj" button. In the new ZoneInfiltration:DesignFlowRate object, input the following (the inputs are also in the partial .idf file):

i. Name: Zone_Infil

ii. Zone or ZoneList Name: ZoneList

iii. Schedule Name: bldg_infil_sched

iv. Design Flow Rate Calculation Method: Flow/ExteriorArea

v. Design Flow Rate $\{\mathrm{m} 3 / \mathrm{s}\}$ : leave blank

vi. Flow per Zone Floor Area $\{\mathrm{m} 3 / \mathrm{s}-\mathrm{m} 2\}$ : leave blank

vii. Flow per Exterior Surface Area $\{\mathrm{m} 3 / \mathrm{s}-\mathrm{m} 2\}$ : 1

viii. Air Changes per Hour $\{1 / \mathrm{hr}\}$ : leave blank

ix. Constant Term Coefficient: 1

x. Temperature Term Coefficient: 0

xi. Velocity Term Coefficient: 0

xii. Velocity Squared Term Coefficient: 0

d. The feature creates one ZoneInfiltration object that applies to the Zone or ZoneList Name "ZoneList". Thus, you need to create a ZoneList object in the EnergyPlus project file that contains the names of all the zones in the EnergyPlus project. Scroll or use the Find function $(\mathrm{Ctrl}+\mathrm{F})$ to go to the ZoneList object (Figure 29). Press the "New Obj" button. In the new ZoneList object, input the following:

i. Name: ZoneList

ii. Zone 1 Name: enter the name of the first zone. You can find a list of all the zones in the EnergyPlus project file in the Zone object, which is right above the ZoneList object. Repeat this for every zone. In the example in Figure 29, there are a total of 5 zones.

If there are existing ZoneList objects in EnergyPlus project file, be sure that none are named "ZoneList". If any are, change the name of the new ZoneList object to anything you choose. Be sure to also copy this name to the ZoneInfiltration:DesignFlowRate object under Zone or ZoneList Name.

Since the ZoneInfiltration object created by this feature specifies that only zones with above-grade exterior surface areas will receive infiltration, the ZoneList can contain the names of interior zones. If there are existing ZoneList objects in EnergyPlus project file, be sure that its name is not "ZoneList". If it is, change the name of the new ZoneList object. Change it also in the ZoneInfiltration:DesignFlowRate object under Zone/ZoneList Name. 


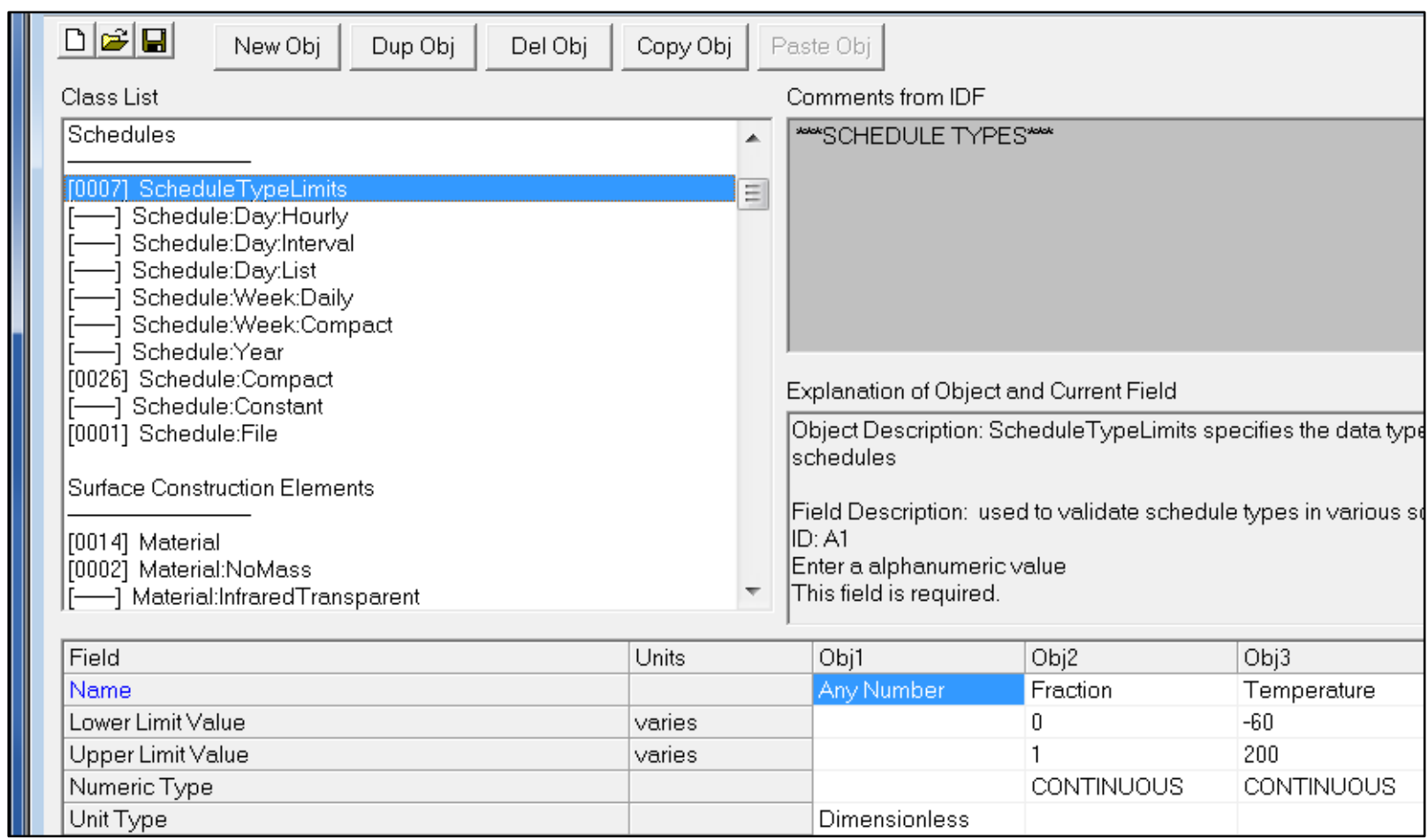

\section{Figure 27. Creating new ScheduleTypeLimits object in EnergyPlus project file}

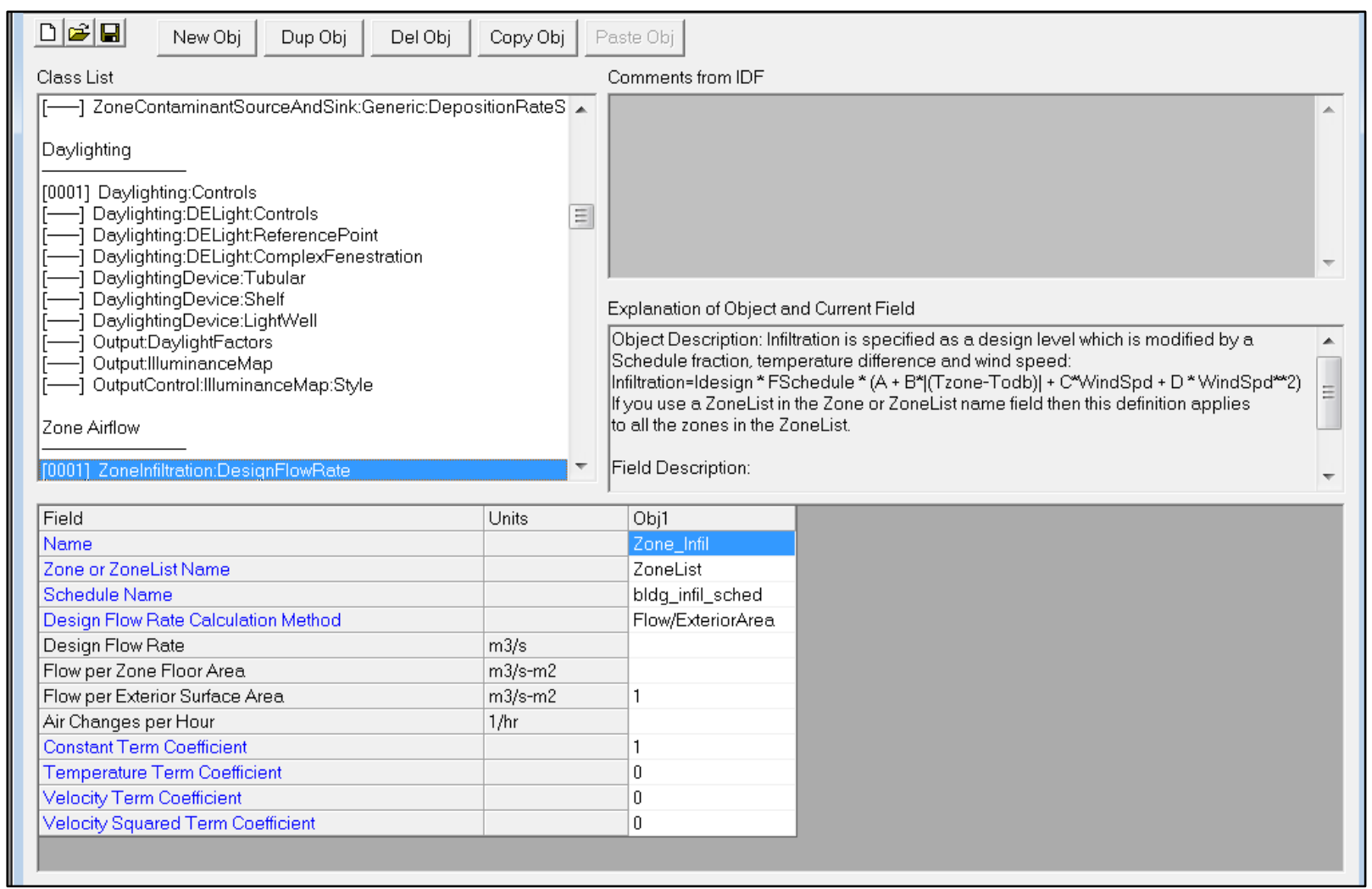

Figure 28. Creating new ZoneInfiltration:DesignFlowRate object in EnergyPlus project file 


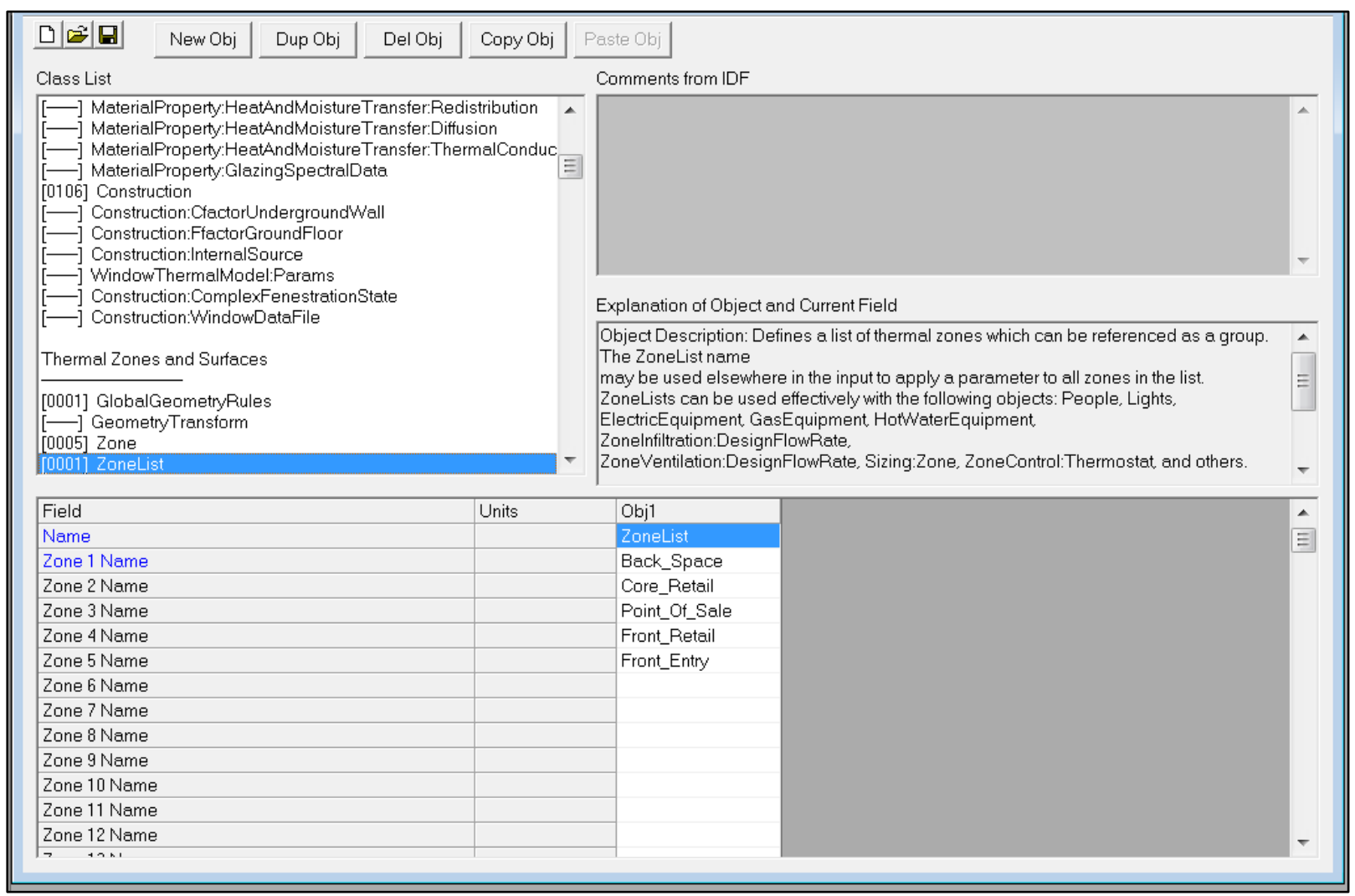

Figure 29. Creating new ZoneList object in EnergyPlus project file 


\section{Feature: Export infiltration to EnergyPlus: zone-specific air change rates}

\subsection{Background}

The background on modeling infiltration in EnergyPlus is the same as in the previous section, Feature: Export infiltration to EnergyPlus: whole-building air change rates. However, the way this feature exports air change rates (zone-specific, as opposed to whole-building in the previous section) for use in EnergyPlus is different.

The feature that exports zone-building air change rates for use in EnergyPlus creates a schedule of $I_{\text {design }}$ values that will be used by EnergyPlus to calculate infiltration rates based on Equation (1), with $A=1$ and $B=C=D=0$. CONTAM infiltration rates are calculated for each zone and exported as flow values $\left(\mathrm{m}^{3} / \mathrm{s}\right)$ using the following equation:

$$
\begin{aligned}
& I_{\text {design,infiltration }}\left(\mathrm{m}^{3} / \mathrm{s} \text { for "zone-specific" feature }\right)=\sum_{j} F_{j i} / \rho \\
& I_{\text {design,exfiltration }}\left(\mathrm{m}^{3} / \mathrm{s} \text { for “zone-specific" feature }\right)=\sum_{j} F_{i j} / \rho
\end{aligned}
$$

where:

$\sum_{j} F_{j i}$ is the sum of outdoor air (zone $j$ ) into the specific zone $i(\mathrm{~kg} / \mathrm{s})$ due only to exterior flow paths calculated by CONTAM.

$\sum_{j} F_{i j}$ is the sum of air leaving the specific zone $i(\mathrm{~kg} / \mathrm{s})$ to the outside due only to exterior flow paths calculated by CONTAM.

$\rho$ is the density of air $\left(\mathrm{kg} / \mathrm{m}^{3}\right)$ calculated by CONTAM.

\subsection{Limitations}

The limitations of this feature are the same as those in the previous section, Feature: Export infiltration to EnergyPlus: whole-building air change rates.

\subsection{What's created by the feature}

This feature will only be available if the option to export detailed building air change rates (.ach) was set via the ContamW simulation output parameters. The ach file contains the wholebuilding air change rates simulated in the .prj file (Dols and Polidoro 2015). This feature will create a partial input data file (partial .idf) file and a comma-separated values (.csv) file.

The partial .idf file contains the following EnergyPlus objects (Figure 30):

- ScheduleTypeLimits - This is a generic limits object that is required by the Schedule:File object. It assigns the values in the Schedule:File object as dimensionless.

- Schedule:File - For each exterior zone, there will be one Schedule:File object created, which calls the column in the .csv file (described below) created by this feature and contains the $I_{\text {design }}$ values associated with a particular zone. 
- ZoneInfiltration:DesignFlowRate - For each exterior zone, there will be one

ZoneInfiltration:DesignFlowRate object, which calls the Schedule:File object that is associated with the same zone. For instance, in Figure 30, the

ZoneInfiltration:DesignFlowRate object named "1_Stock_infil" calls the Schedule:File object named "1_Stock_infilt_sched", which then calls the second column of the "example1.csv" file that was created by this feature.

The .csv file is created using the CONTAM infiltration rates. The columns in the .csv file are: timestamps, and alternating columns of infiltration and exfiltration values for each exterior zone (Figure 31). Even though the exfiltration is exported using this feature, the

ZoneInfiltration:DesignFlowRate objects are set so that EnergyPlus only calls the infiltration rates during the simulation. The .csv file needs to be located in the same directory as the EnergyPlus project file. Step-by-step instructions on how to use these files in an EnergyPlus project will be given next.

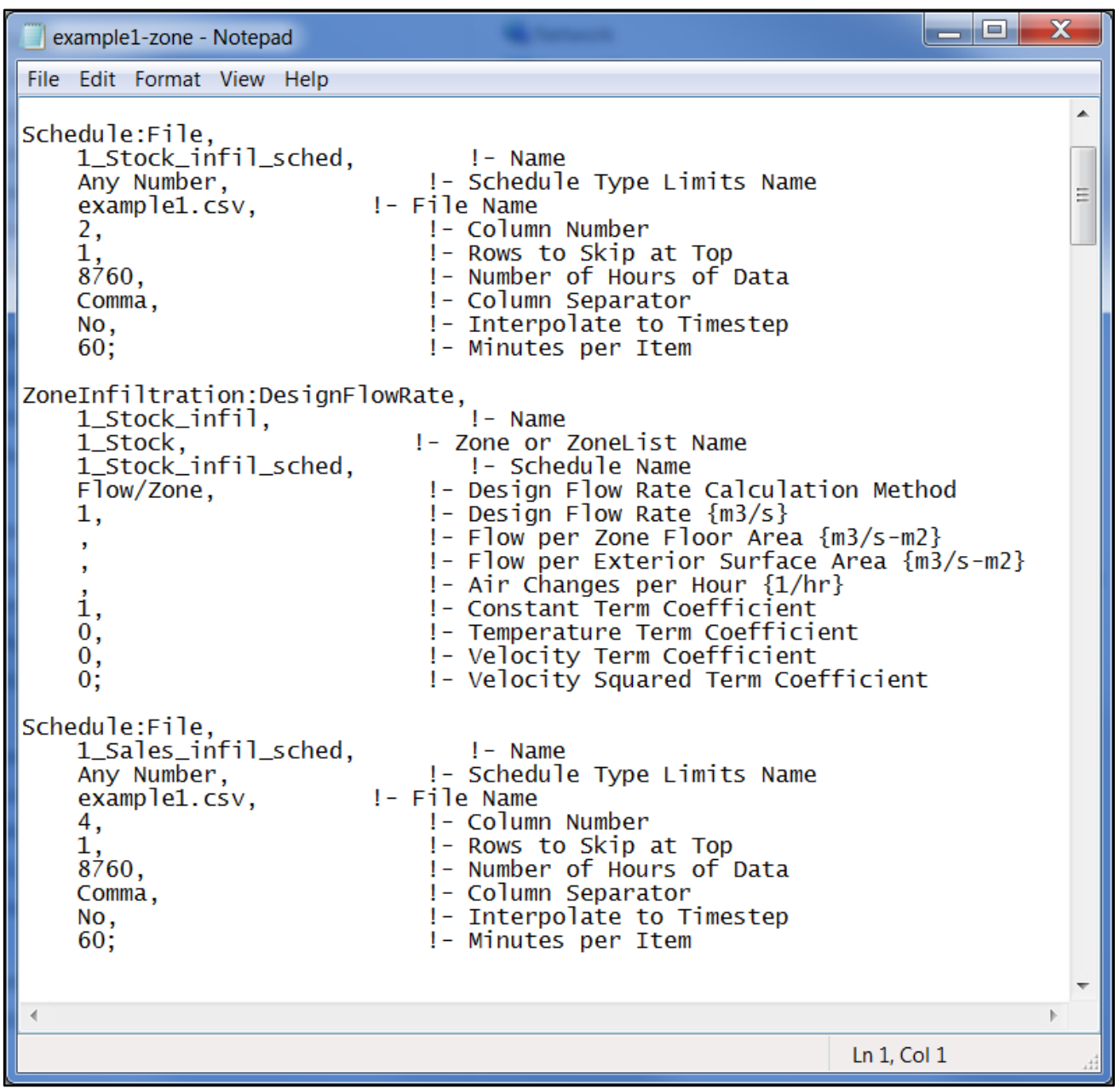

Figure 30. Partial .idf file created by "Export infiltration to EnergyPlus: zone-specific air change rates" feature 


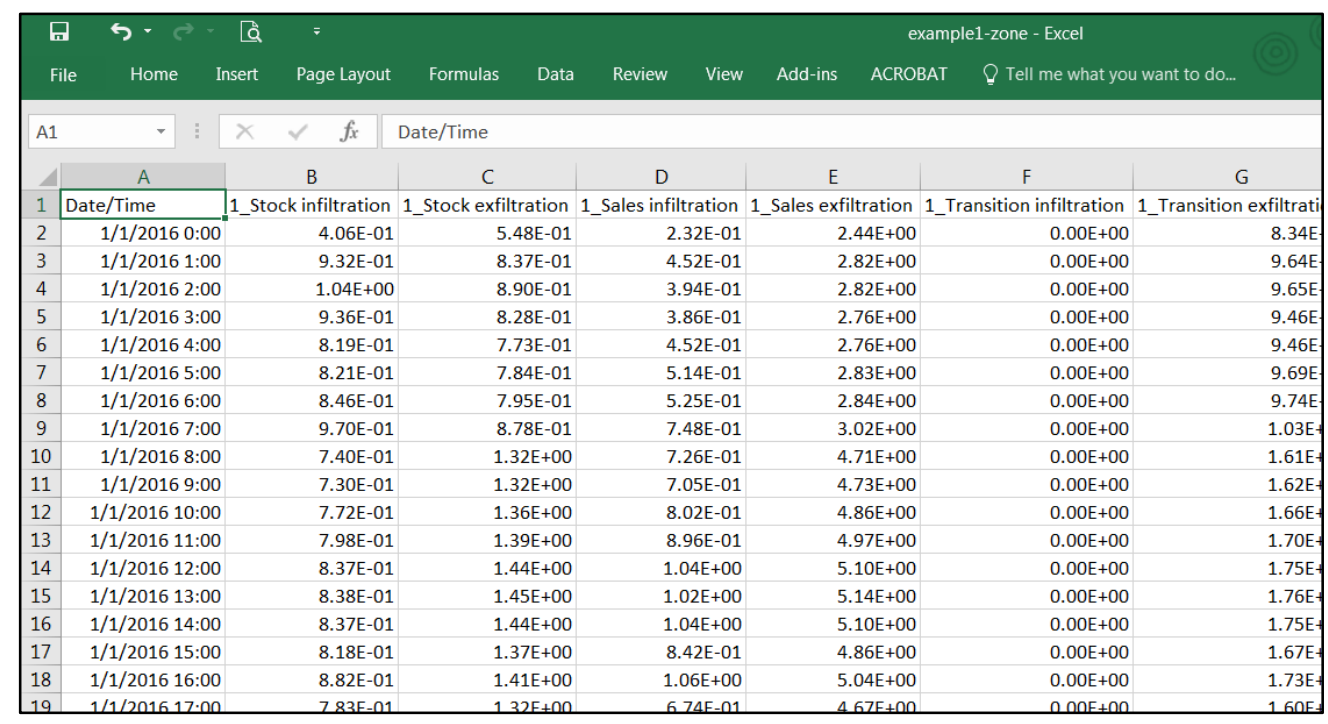

Figure 31. CSV file created by "Export infiltration to EnergyPlus: zone-specific air change rates" feature

\subsection{Using the feature}

Figure 32 presents a flow diagram of the steps involved in exercising this feature. The numbered/colored boxes refer to figure numbers throughout the step-by-step instructions that follow and that show screen shots of the Export Tool.

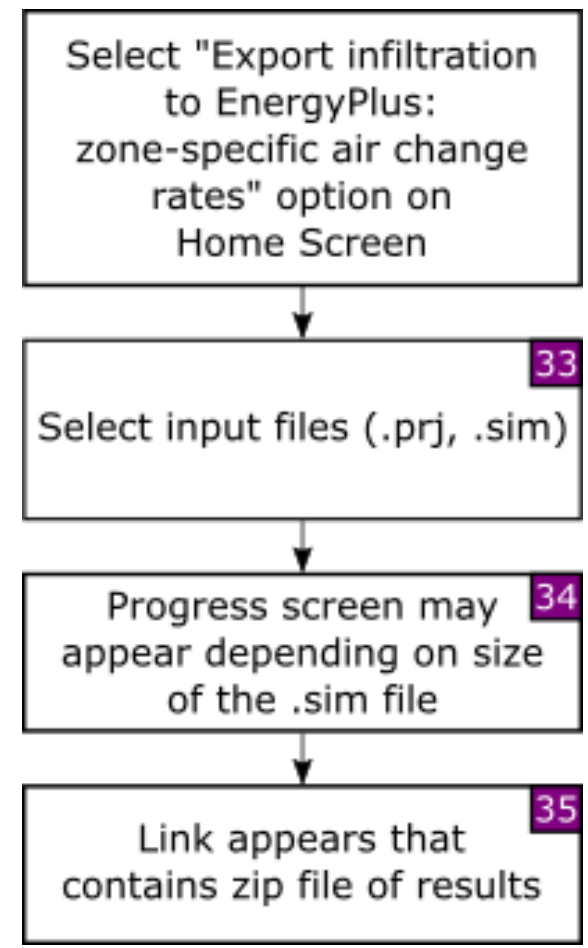

Figure 32. Flow diagram of the "Export infiltration to EnergyPlus: zone-specific air change rates" feature 
7.5. Step-by-step instructions for the "Export infiltration to EnergyPlus: zonespecific air change rates" feature

1. On the CONTAM Results Export Tool Home Screen (Figure 1), select the "Export infiltration to EnergyPlus: zone-specific air change rates" radio button then click the "Next" button.

2. Select both a CONTAM project (.prj) and simulation results (.sim) file (Figure 33).

3. A progress screen may appear, depending on the size of the .sim file (Figure 34).

4. Once the export is complete, a link will be provided to a compressed zip file (Figure 35) that contains the partial idf and .csv files, which contains the zone-specific air change rates and EnergyPlus input objects. The use of these files in an EnergyPlus simulation will be described next. Right-click on the link to either download the zip file to your computer, or to open the zip file and copy extracted the file(s) to your computer.

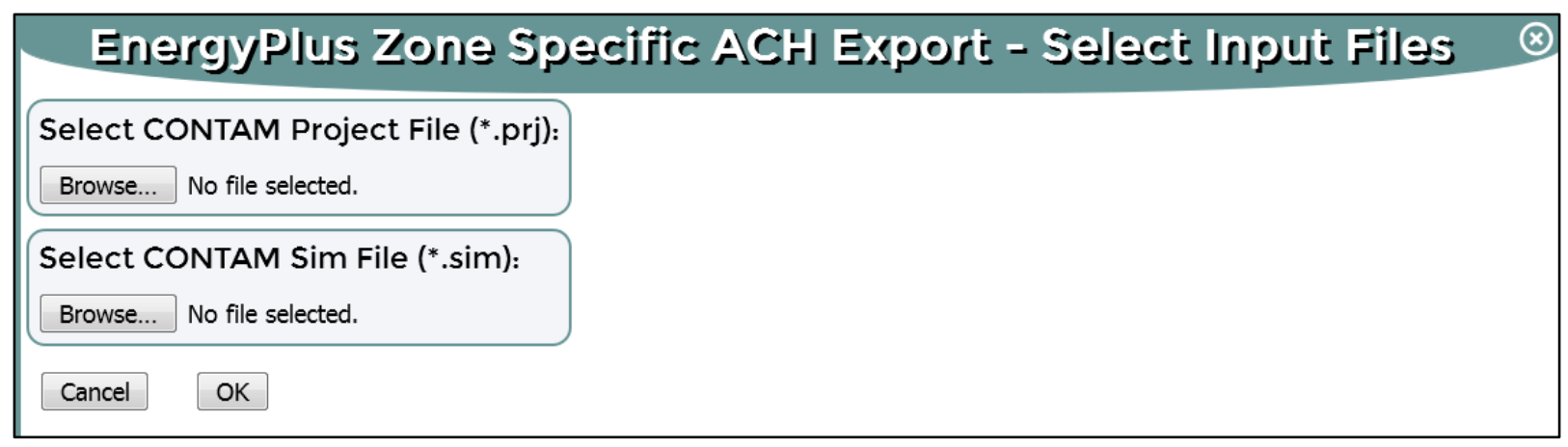

Figure 33. Selecting CONTAM project (.prj) and simulation results (.sim) files

\begin{tabular}{|l|}
\hline EnergyPlus ZZone Specific ACH Export - Progress \\
\hline Date: $1 / 7$ \\
\hline Time: $16: 00: 00$ \\
\hline
\end{tabular}

Figure 34. Progress of exporting zone-specific air change rates

$$
\text { Energyplus Żone Specific ACH Export - Save Files }
$$

Save zip file with EnergyPlus Export files

Figure 35. Exporting zone-specific air change rates (link containing zip files) 


\subsection{What to do with the .csv and partial .idf files}

Figure 36 presents a flow diagram of the steps to utilize the files generated by the feature in an EnergyPlus simulation. The numbered/colored boxes refer to figure numbers throughout the step-by-step instructions that follow and that show screen shots of the text editor and EnergyPlus IDF Editor program.

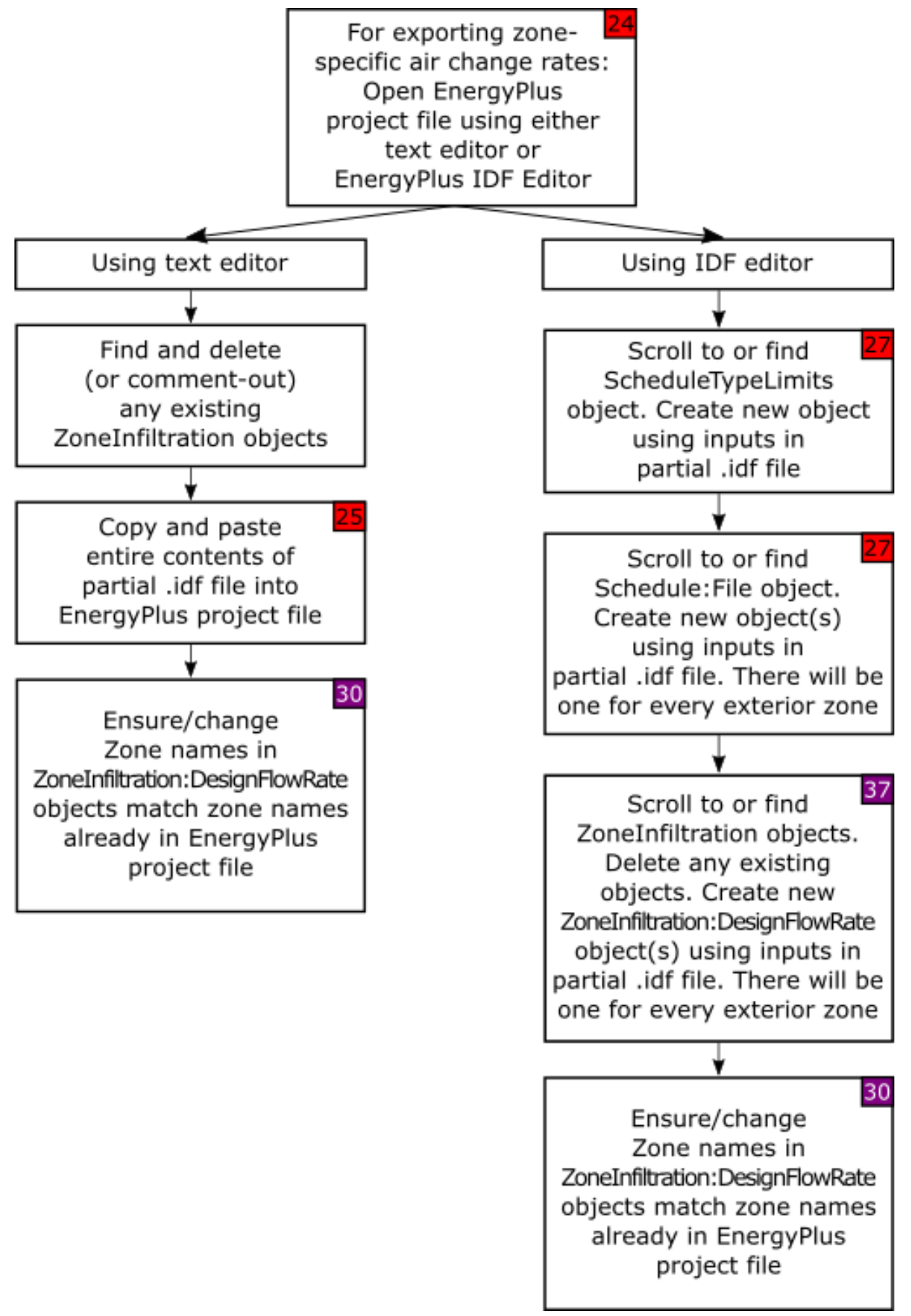

Figure 36. Flow diagram of what to do with the files generated by the "Export infiltration to EnergyPlus: zone-specific air change rates" feature 
1. Open the EnergyPlus project file, into which you want to import CONTAM infiltration rates, with either a text editor or the EnergyPlus IDF Editor program (Figure 24).

2. If using a text editor:

a. Use the Find function $(\mathrm{Ctrl}+\mathrm{F})$ to locate any existing ZoneInfiltration objects. Delete any existing ZoneInfiltration objects or make them comment lines by adding an exclamation point (!) at the beginning of each line.

b. Copy and paste the entire contents of the partial idf created by this feature into the EnergyPlus simulation file. It does not matter where, however do not break-up any existing EnergyPlus objects by pasting the text from the partial idf file below an input that ends in a semicolon (Figure 25). If you would like similar objects to be grouped together, you can open up the updated EnergyPlus simulation file in the EnergyPlus IDF Editor program. When you use the "Save as" function, the objects in the EnergyPlus simulation file will re-order the objects and group similar objects together.

c. The feature creates one ZoneInfiltration:DesignFlowRate object for each exterior zone (Figure 30). The Name of each ZoneInfiltration:DesignFlowRate object follows the naming convention "CONTAM level number" "CONTAM zone name"_infil. The associated Zone or ZoneList Name follows the naming convention "CONTAM level number" "CONTAM zone name". Thus, you need to ensure that the Zone or ZoneList Names match the zone names in the EnergyPlus project file. You can find a list of all the zones in the EnergyPlus project file.

If the CONTAM model has more exterior zones than in the EnergyPlus model, multiple ZoneInfiltration:DesignFlowRate objects can be applied to the same zone in EnergyPlus. It is up to the user to decide which EnergyPlus zones will be assigned multiple ZoneInfiltration:DesignFlowRate objects, if needed.

\section{If using the EnergyPlus IDF Editor program:}

a. Scroll or use the Find function $(\mathrm{Ctrl}+\mathrm{F})$ to go to the ScheduleTypeLimits object (Figure 27). Press the "New Obj" button. In the new ScheduleTypeLimits object, input the following (the inputs are also in the partial .idf file):

i. Name: Any Number

ii. Unit Type: Dimensionless

b. Scroll or use the Find function $(\mathrm{Ctrl}+\mathrm{F})$ to go to the Schedule:File object (Figure 27). Press the "New Obj" button. In the new Schedule:File object, input the following (the inputs are also in the partial idf file):

i. Name: copy the name of the first Schedule:File object in the partial idf file. For example, Figure 30 shows that first Schedule:File object name to be "1_Stock_infil_sched".

ii. Schedule Type Limits Name: Any Number

iii. File Name: copy the name of the .csv file created by this feature. It is also in partial .idf file. For example, Figure 30 shows that filename is "example1.csv".

iv. Column Number: copy the column number associated with the Schedule:File object. Continuing the example, Figure 30 shows this to be " 2 ". 
v. Rows to Skip at Top: 1

vi. Number of Hours of Data: 8760

vii. Column Separator: Comma

viii. Interpolate to Timestep: No

ix. Minutes per Item: 60

Repeat this for every Schedule:File object in the partial idf file created by this feature.

c. Scroll or use the Find function $(\mathrm{Ctrl}+\mathrm{F})$ to go to the ZoneInfiltration:DesignFlowRate object (Figure 37). Delete any existing ZoneInfiltration:DesignFlowRate objects. Press the "New Obj" button. In the new ZoneInfiltration:DesignFlowRate object, input the following (the inputs are also in the partial .idf file):

i. Name: copy the name of the first ZoneInfiltration:DesignFlowRate object in the partial .idf file. For example, Figure 30 shows that first ZoneInfiltration:DesignFlowRate object name to be "1_Stock_infil".

ii. Zone or ZoneList Name: copy the Zone or ZoneList Name associated with the ZoneInfiltration:DesignFlowRate object. Continuing the example, Figure 30 shows this to be "1_Stock".

iii. Schedule Name: copy the Schedule Name associated with the ZoneInfiltration:DesignFlowRate object. Continuing the example, Figure 30 shows this to be "1_Stock_infil_sched".

iv. Design Flow Rate Calculation Method: Flow/Zone

v. Design Flow Rate $\{\mathrm{m} 3 / \mathrm{s}\}$ : 1

vi. Flow per Zone Floor Area $\{\mathrm{m} 3 / \mathrm{s}-\mathrm{m} 2\}$ : leave blank

vii. Flow per Exterior Surface Area $\{\mathrm{m} 3 / \mathrm{s}-\mathrm{m} 2\}$ : leave blank

viii. Air Changes per Hour $\{1 / \mathrm{hr}\}$ : leave blank

ix. Constant Term Coefficient: 1

x. Temperature Term Coefficient: 0

xi. Velocity Term Coefficient: 0

xii. Velocity Squared Term Coefficient: 0

Repeat this for every ZoneInfiltration:DesignFlowRate object in the partial idf file created by this feature.

The feature creates one ZoneInfiltration:DesignFlowRate object for each exterior zone (Figure 30). Thus, you need to ensure that the Zone or ZoneList Names match the zone names in the EnergyPlus project file. You can find a list of all the zones in the EnergyPlus project file.

If the CONTAM model has more exterior zones than in the EnergyPlus model, multiple ZoneInfiltration:DesignFlowRate objects can be applied to the same zone in EnergyPlus. It is up to the user to decide which EnergyPlus zones will be assigned multiple ZoneInfiltration:DesignFlowRate objects, if needed. 


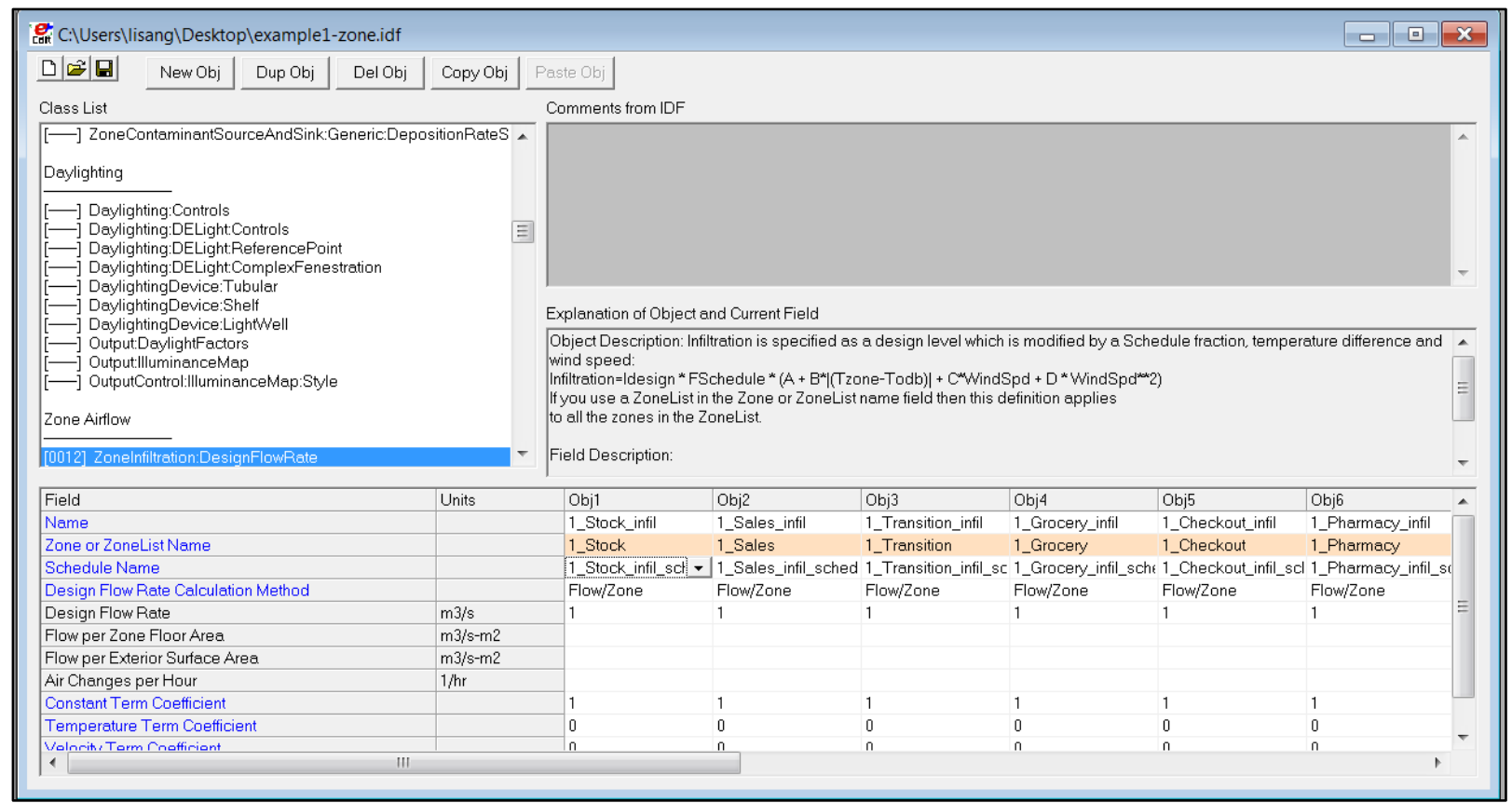

Figure 37. Creating new ZoneInfiltration:DesignFlowRate object(s) in EnergyPlus project file 


\section{Conclusion}

The CONTAM Results Export Tool provides a flexible means to extract result from the wide range of data that is generated by CONTAM simulations. The results that can be exported include contaminant concentrations, zone temperatures, weather conditions, airflow from paths and duct leakages, temporal and spatial averages of concentration, occupant exposure, and exporting CONTAM infiltration results for use in EnergyPlus simulations.

\section{References}

DOE (2013). EnergyPlus 8.2. Washington, D. C., U. S. Department of Energy.

Dols, W. S. and B. Polidoro (2015). CONTAM User Guide and Program Documentation.

Technical Note 1887. Gaithersburg, MD: National Institute of Standards and Technology.

Ng, L. C., S. J. Emmerich and A. K. Persily (2014). An Improved Method of Modeling Infiltration in Commercial Building Energy Models. Technical Note 1829. Gaithersburg, MD: National Institute of Standards and Technology.

Walton, G. N. (1989). AIRNET - A Computer Program for Building Airflow Network Modeling. NISTIR 89-4072. Gaithersburg, MD: National Institute of Standards and Technology. 